\title{
Gustave Le Bon (1923)
}

\section{Les incertitudes \\ de l'heure présente}

\section{Réflexions sur la politique, les guerres, les alliances, la vie, le droit, la morale, les religions, les philosophies, etc.}

Un document produit en version numérique par Roger Deer, bénévole, ingénieur à la retraite, diplômé de l'ENSAIA de Nancy

(école nationale supérieure d'agronomie et de industries alimentaires)

Courriel : roger.derr@wanadoo.fr

Dans le cadre de la collection : "Les classiques des sciences sociales" fondée et dirigée par Jean-Marie Tremblay, professeur de sociologie

Site web : http://www.uqac.uquebec.ca/zone30/Classiques_des_sciences_sociales/index.html

Une collection développée en collaboration avec la Bibliothèque

Paul-Émile-Boulet de l'Université du Québec à Chicoutimi

Site web : http ://bibliotheque.uqac.uquebec.ca/index.htm 
Cette édition électronique a été réalisée par Roger Deer, bénévole, ingénieur à la retraite, diplômé de l'ENSAIA de Nancy (école nationale supérieure d'agronomie et de industries alimentaires) roger.derr@wanadoo.fr

à partir de :

\section{Gustave Le Bon (1923)}

\section{Les incertitudes de l'heure présente.}

Réflexions sur la politique, les guerres, les alliances, la vie, le droit, la morale, les religions, les philosophies, etc.

Une édition électronique réalisée à partir du livre de Gustave Le Bon, Les incertitudes de l'heure présente (1923) Réflexions sur la politique, les guerres, les alliances, la vie, le droit, la morale, les religions, les philosophies, etc. Paris: Ernest Flammarion, Éditeur, 1923, 230 pages.

Polices de caractères utilisée :

Pour le texte : Times, 12 points.

Pour les citations : Times 10 points.

Pour les notes de bas de page : Times, 10 points.

Édition électronique réalisée avec le traitement de textes Microsoft Word 2001 pour Macintosh.

Mise en page sur papier format

LETTRE (US letter), 8.5' x 11'')

Mise en page complétée le 13 mars 2003 à Chicoutimi, Québec. 


\section{Table des matières}

Remarque sur la présente édition numérique, par Roger Deer

Dédicace

Préface

Chapitre I : $\quad$ La vie politique

I. Perturbations politiques et morales créées par la guerre

II. Les Difficultés modernes des Gouvernements

III. Les Croyances politiques

IV. Les Formules politiques

V. Les Erreurs de psychologie en politique

Chapitre II : $\quad$ Les guerres, les révolutions et le désarmement

I. Les Causes futures de guerres et la Revanche germanique

II. Les Luttes pour l'hégémonie

III. Les Illusions sur la possibilité d'un désarmement

IV. Les Incertitudes sur les origines de la guerre

V. Les Causes des Révolutions

VI. Les Résultats des Révolutions

Chapitre III : $\quad$ Les relations internationales et les alliances

I. Les Relations internationales

II. Les Forces économiques

III. Les Traités de paix et les grands Congrès politiques

IV. Les Traités d'alliance et leur valeur

V. La Société des Nations

Chapitre IV : Le droit et la morale

I. Les Coutumes et les Lois

II. Le Droit et la Force

III. Les Forces morales

IV. Les Sources de la Morale

Chapitre V : $\quad$ Les formes modernes du despotisme

I. L'Extrémisme

II. Le Socialisme

III. Le Syndicalisme

IV. Le Communisme.

V. L’Égalité et le Besoin de servitude. 
Chapitre VI : $\quad \underline{\text { L'évolution des civilisations }}$

I. Comment les Civilisations naissent et pourquoi elles disparaissent

II. Les Institutions politiques

III. Quelques Conséquences des idées démocratiques.

IV. Les Récits historiques

Chapitre VII : L'intelligence, le caractère et l'éducation

I. L'incompréhension et les conflits de mentalités.

II. Le Caractère et l'Intelligence dans la vie des Peuples

III. L'Intelligence, les Sentiments et l'Intuition

IV. L'instruction et l'Éducation

Chapitre VIII : Les influences conscientes et inconscientes dans la vie des peuples

I. La Vie consciente et la Vie inconsciente

II. La Vie collective. Les Meneurs et les Menés

III. L'Âme des peuples

IV. Les Oscillations de l'opinion

Chapitre IX : Les dieux dans l'histoire

I. Le Rôle des Dieux

II. Le Pouvoir des croyances

III. Les Formes diverses des croyances

IV. La Raison et la Foi

Chapitre X: Visions philosophiques du monde

I. Les Conceptions philosophiques du Monde

II. La Vérité et la Certitude

III. Les Lois de la Vie

IV. La Sagesse et le Bonheur

V. L'imprévisible et la Région des causes 


\section{Remarque sur cette édition numérique}

Par Roger Deer

12 mars 2003

$\underline{\text { Retour à la table des matières }}$

L'ouvrage a été publié en 1923, l'exemplaire dont je dispose appartient à l'édition originale. J'ignore si des rééditions ont eu lieu par la suite.

Les fautes sont très rares. Comme d'habitude on trouve "déclancher" à plusieurs reprises :

page 9 : déclanchement (corrigé)

page 43 : déclanché (corrigé)

page 133 : déclanchèrent (corrigé)

page 198 : crocrodile (corrigé)

mais c'est tout il n'y a pas d'autres fautes, il faut dire que l'ouvrage est court. 


\title{
À \\ MON AMI \\ ARISTIDE BRIAND \\ ANCIEN PRE5IDEMT DU CONSEIL DES MINISTRES
}

\author{
En souvenir \\ de nos longues causeries philosophiques \\ durant les pesantes années de guerre \\ et les heures incertaines qui la suivent.
}

GUSTAVE LE BON.

$\underline{\text { Retour à la table des matières }}$ 
Les incertitudes de l'heure présente

\section{Préface}

$\underline{\text { Retour à la table des matières }}$

$-\mathrm{I}-$

L'évolution scientifique moderne a fait naître des nécessités économiques nettement contraires aux impulsions affectives et mystiques qui, depuis les débuts de l'histoire, dirigent les actions des hommes.

Cette opposition, accentuée chaque jour, est une des causes profondes du déséquilibre actuel. Notre époque oscille entre les influences héréditaires qui orientaient jadis le monde et les nécessités issues des découvertes scientifiques nouvelles.

Comment concilier les ambitions, les rivalités et les haines poussant les races à de furieuses luttes, avec l'engrenage économique qui les lie d'une si étroite interdépendance que le dommage subi par l'une d'elles atteint bientôt toutes les autres ?

-II-

Si cette interdépendance n'a pas réussi à faire de la solidarité une des lois du monde moderne, c'est que les passions et les sentiments, générateurs habituels de la conduite, sont l'héritage d'un long passé, alors que les nécessités économiques nouvelles, datant d'hier, pèsent peu encore dans la balance des motifs qui font agir les hommes. 
La domination des forces rationnelles par les forces affectives et mystiques doit être toujours présente à l'esprit quand on veut comprendre la genèse des grands événements qui perturbent la vie des peuples.

Croire ces événements déterminés par la pure logique rationnelle conduit à de redoutables illusions.

Ils en furent victimes, les pacifistes qui, à la veille de la guerre, soutenaient, avec un éminent professeur de la Sorbonne, qu'un conflit entre la France et l'Allemagne étant rationnellement impossible, une coûteuse préparation militaire devenait inutile.

Les faits leur prouvèrent bientôt que la logique savante des professeurs ne régit pas encore l'Histoire. Les logiques affective et mystique qui l'orientent obéissent à de tout autres lois. Nous aurons plus d'une fois à en marquer la nature dans cet ouvrage.

-III-

Les réflexions provoquées par les agitations de l'heure présente varient naturellement suivant les habitudes mentales de l'observateur. Les points de vue du savant ne sauraient être ceux du croyant dont la foi limite l'horizon, ni ceux de l'homme d'État absorbé par les nécessités journalières, moins encore ceux des adeptes d'un parti politique, uniquement préoccupés des intérêts de ce parti.

S'élever au-dessus de ces barrières est nécessaire pour percevoir les origines et les conséquences des problèmes qui troublent si profondément aujourd'hui l'âme des nations.

Dans l'état présent de nos connaissances, et après les bouleversements qui ont ébranlé l'antique armature sociale, quelles idées peut-on se faire du droit, de la morale, des institutions, des croyances religieuses, politiques et sociales qui ont guidé la marche des civilisations et la guident encore ?

$-\mathrm{IV}-$

Une réponse suffisante à de telles questions exigerait des volumes. Mais les phénomènes sociaux, de même d'ailleurs que les phénomènes physiques, sont dominés, malgré leur complexité, par quelques principes fondamentaux dont les cas particuliers découlent et qu'il est possible de formuler brièvement. Ces principes, véritable substratum des choses, sont plus suggestifs, souvent, que de longues explications.

C'est pourquoi je me suis décidé une fois encore à condenser en pensées brèves les observations dérivées des grands événements qui troublent notre époque.

Les vues d'un philosophe ayant beaucoup exploré le monde et recherché aussi des vérités nouvelles dans le silence des laboratoires, présenteront peut-être quelque intérêt. 
Dégagées des passions trompeuses et des récriminations stériles, elles pourront contribuer à dissiper les brumes qui obscurcissent le présent et rendent si incertain l'avenir. 
Les incertitudes de l'heure présente

\section{Chapitre I}

\section{La vie politique}

\section{1 - Perturbations politiques et morales créées par la guerre}

$\underline{\text { Retour à la table des matières }}$

L'instabilité universelle est une des plus visibles conséquences de la guerre instabilité des institutions, instabilité des alliances, instabilité des pensées.

Ce n'est pas seulement l'Europe matérielle, mais l'Europe morale, qu'il faudrait pouvoir reconstruire. Cependant, les ambitions, les haines et les besoins grandissent, alors que le goût du travail, la discipline et le sentiment du devoir ne cessent de faiblir.

Toutes les anciennes armatures sociales ayant été ébranlées par la conflagration universelle, les peuples cherchent à tâtons des institutions nouvelles. S'ils reviennent invariablement aux anciennes, c'est probablement qu'il n'en existe pas d'autres. 
Les équilibres d'États formés avant la guerre étaient stables parce qu'ils avaient mis des siècles à se former. Les équilibres artificiels créés depuis la paix sont instables parce qu'ils dérivent de principes théoriques, étrangers aux réalités.

L'Europe marche visiblement vers de nouveaux groupements politiques qui ne seront ni ceux antérieurs à la guerre, ni ceux fondés par elle. L'Italie s'oriente vers l'Angleterre, l'Allemagne vers l'Angleterre et la Russie, la France vers la Turquie, la Pologne et les États balkaniques. Des luttes nombreuses deviendront nécessaires pour stabiliser ces nouveaux équilibres.

La ruine des classes intellectuelles moyennes et leur retour forcé à un demiprolétariat coïncidant avec l'aisance des anciens prolétaires est une des plus dangereuses conséquences de la guerre.

Le nombre des soldats victimes de la grande guerre est connu. Celui des idées et des croyances détruites par elle reste encore ignoré.

Parmi les causes profondes du déséquilibre social actuel, figure la perte partielle des habitudes mentales qui orientaient jadis la conduite et dispensaient d'avoir trop à réfléchir avant d'agir.

Quand les idées qui soutiennent une société s 'écroulent, cette société tombe dans l'anarchie jusqu'au jour où elle retrouve d'autres principes directeurs assez puissants pour lui constituer une nouvelle armature. Tout changement d'idéal impliquant de profonds bouleversements, le passage d'un idéal à un autre est toujours fort long.

\section{II - Les difficultés modernes des gouvernements}

$\underline{\text { Retour à la table des matières }}$

La vérité, pour la grande majorité des hommes, étant ce qu'ils croient, c'est surtout avec leurs croyances qu'on doit gouverner les peuples.

Parmi les connaissances psychologiques les plus nécessaires aux gouvernants figure l'art de pénétrer la mentalité d'hommes dont les idées diffèrent des leurs et de raisonner avec ces idées. 
Une des graves difficultés de la politique est l'obligation de gouverner avec des idées tenues pour vraies par les multitudes alors que ces idées sont erronées.

Jadis, les hommes d'État gouvernaient en s'appuyant sur des coutumes et des traditions très fixes. Obligés aujourd'hui de suivre des convictions populaires mobiles, ils doivent se borner à suggérer des opinions acceptables et essayer de modifier celles qui ne le sont pas.

Un pays peut changer de gouvernement mais ses traditions politiques ne changent guère. La Convention a continué sur bien des points la politique de Louis XIV. Les bolchevistes eux-mêmes poursuivent en Orient celle des tsars.

Les gouvernants doivent savoir discerner les sentiments qui font mouvoir les hommes, sans se préoccuper beaucoup des influences rationnelles qui devraient les faire agir.

Quel que soit le mode de gouvernement, il aboutit toujours à une oligarchie permanente dans le régime monarchique, éphémère dans le régime démocratique.

Les gouvernants doivent savoir ce qu'ils veulent et ce qu'ils peuvent, mais aussi ce que veulent et peuvent leurs adversaires.

Bien connaître les bornes de son pouvoir est nécessaire afin de ne jamais s'approcher des limites où se manifesterait l'impuissance.

Le déclenchement des événements appartient souvent aux hommes d'État, mais devant leur déroulement ils restent impuissants. L'Allemagne pouvait décider ou ne pas décider la guerre sous-marine à outrance une fois commencée, elle entraînait fatalement l'intervention de l'Amérique. Le premier ministre anglais conseillant aux Polonais de traiter avec l'armée bolcheviste qui cernait leur capitale, préparait l'invasion de l'Europe, s'il eût été écouté.

Dans les pays composés de peuples différant par la religion, la race et la langue, un régime despotique est seul capable d'empêcher de sanguinaires luttes intestines. Jamais les Balkaniques ne se sont autant massacrés que depuis leur affranchissement de la domination turque. Elle seule avait réussi à maintenir la paix parmi eux pendant cinq cents ans.

Reculer devant un danger a pour résultat certain de le grandir.

Ne pas résister, en politique, à une force antagoniste naissante est se condamner à la voir devenir irrésistible. Les Girondins de tous les âges en ont fait l'expérience. 
Cette éternelle loi conduisit la révolution russe, d'abord pacifique, à sombrer dans une sanglante dictature.

Les apôtres ne se combattant qu'avec des apôtres, on ne triomphe des meneurs qu'en leur opposant d'autres meneurs.

Un ministre ne saurait être le même homme au pouvoir et hors du pouvoir. Au pouvoir, il s'occupe nécessairement des intérêts généraux. Hors du pouvoir, il perçoit seulement ses intérêts personnels, dont le plus essentiel est de remonter au pouvoir.

Entre hommes politiques de partis différents l'amitié est possible. Entre hommes d'un même parti la jalousie est généralement trop intense pour permettre l'amitié.

Si la carrière diplomatique exigeait un examen prouvant la connaissance du caractère des divers peuples, de leurs réactions possibles suivant les circonstances et des moyens d'influencer efficacement leur conduite, on ne trouverait sans doute pas dix hommes en Europe capables de bien passer cet examen.

Une science approfondie des choses paralyse souvent l'action. Des hommes d'État possédant un esprit assez vaste pour percevoir toutes les conséquences possibles de leurs décisions agiraient fort peu.

L'homme d'État capable de prévoir toutes, les répercussions, de ses actes serait comparable au joueur d'échecs lisant sur l'échiquier de son adversaire les possibilités invisibles résultant du déplacement des pièces visibles.

La vie politique et sociale n'étant possible qu'au moyen de transactions et de compromis, l'intransigeance constitue la plus dangereuse des doctrines.

Un gouvernement quelconque est toujours entouré de forces hostiles. L'habileté consiste à les orienter pour n'avoir pas à les combattre.

En politique, il est à peu près impossible de juger avec équité les opinions d'un adversaire.

Les hommes d'État font bien d'utiliser la logique rationnelle dans leurs discours, mais ils ne doivent jamais oublier que les peuples sont souvent conduits par des passions, des croyances, des intérêts fort étrangers à la logique des livres.

Pour qu'une menace politique conserve son prestige, il faut reculer le plus possible sa réalisation. 
Le temps aide les gouvernements forts, mais rarement les gouvernements faibles.

Les maîtres des peuples n'ont pas seulement à régir les vivants, Il leur faut tenir compte aussi de l'impérieuse volonté des morts.

\section{III - Les croyances politiques}

$\underline{\text { Retour à la table des matières }}$

Croyances religieuses et croyances politiques ont des fondements psychologiques identiques. Elles naissent et se propagent de la même façon.

La propagation de certaines croyances politiques, telles que le communisme, est incompréhensible quand on ignore le mystique besoin de croyance qui domine la vie des peuples.

Les vérités scientifiques sont des vérités universelles. Les croyances politiques, tenues pour des vérités, représentent habituellement des convictions transitoires, issues de passions et de sentiments que la raison ne gouverne pas.

Alors même que les religions semblent ne plus agir sur les âmes, leur puissance se maintient dans l'inconscient et reste mobile d'action. La haine contre la Turquie, si énergiquement manifestée par les Anglais et les Américains, représente une survivance ancestrale de la lutte séculaire entre la Croix et le Croissant.

Une croyance politique n'est, souvent, qu'un acte de foi dépourvu de support rationnel. Elle a pour origine le mécontentement chez les illettrés, l'envie et l'ambition chez les hommes instruits.

Si destructive que soit une croyance politique, elle trouve toujours pour la défendre des intellectuels dont les ambitions dépassaient les capacités.

Certaines croyances politiques attirent une foule de rhéteurs totalement indifférents à ces croyances, mais espérant les utiliser au profit de leurs convoitises. Catilina vivant aujourd'hui se déclarerait syndicaliste ou bolchéviste. 
Une croyance rationnellement fausse, mais capable de solidariser les hommes, est politiquement supérieure à une doctrine rationnellement exacte, mais impuissante à créer l'unité de pensée sans laquelle les peuples ne peuvent prospérer. C'est à la lumière de ce principe que l'histoire des croyances politiques et religieuses doit se juger.

On se représente difficilement l'existence d'un peuple gouverné par des réalités au lieu de l'être par des illusions religieuses, politiques ou sociales. L'histoire n'en cite pas d'exemple.

Dans les cycles de l'affectif et du mystique, où s'élaborent les croyances politiques et sociales, l'intelligence pénétrant fort peu, les convictions des illettrés et des savants n'ont pas une valeur bien différente.

L'exemple du dictateur bolcheviste envoyant du Kremlin ses ordres aux révolutionnaires respectueusement réunis en congrès à Tours pour les recueillir, contribue à montrer combien le besoin de soumission à des dogmes reste intense même chez des révoltés s'imaginant libérés de toute croyance.

Dès qu' elles atteignent un certain degré, les croyances mystiques, religieuses ou politiques, deviennent fatalement destructives.

En art comme en politique, le prestige est un grand régulateur des valeurs. Lorsque le Louvre achetait 700.000 francs un tableau qui lui avait été offert pour 20.000 quelques années auparavant, il payait simplement le prestige acquis par le nom de l'auteur. La valeur numérique de ce prestige était exactement représentée par la différence entre les deux sommes. Le prestige des formules politiques subit souvent des variations du même ordre.

Les progrès du bolchevisme contribuent à prouver qu'une doctrine chargée d'espérances s'impose plus facilement que les vérités rationnelles les mieux démontrées.

Républicains et socialistes constituent. malgré leur collaboration éphémère, deux partis politiques opposés. Les premiers représentent la démocratie, les seconds la dictature.

Une des forces du convaincu est de ne pas discuter la valeur rationnelle de sa croyance. 
En politique et en religion, le rêve des convaincus fut toujours de pouvoir massacrer sans pitié les hommes qui ne pensent pas comme eux.

Une excellente définition du radicalisme est celle qu'en donna jadis le président Wilson. "Ce terme signifie, disait-il simplisme, violence et envie."

Le radicalisme durera longtemps sans doute, parce que la nature humaine suppose volontiers que des mesures simples et violentes peuvent remédier instantanément à des maux résultant, en réalité, d'un ensemble de causes lointaines et profondes.

Lorsque les raisons psychologiques des événements et leur complication seront mieux comprises, bien peu d'hommes instruits consentiront à se dire radicaux. répétée.

En politique, une vérité indiscutée n'est souvent qu'une erreur suffisamment

\section{IV - Les formules politiques}

$\underline{\text { Retour à la table des matières }}$

Dans les sciences, la valeur d'une idée demeure indépendante des formules qui la traduisent. En politique ce sont uniquement les formules qui agissent sur les multitudes.

Dans les assemblées politiques, le prestige du verbe domine généralement la compétence.

Une idée n'acquiert d'influence qu'après être devenue collective. Elle s'extériorise alors en formules, et peut devenir assez forte pour orienter la vie d'un peuple.

Une formule bien choisie est capable de bouleverser le monde. Simple, brève et violente, elle impressionne beaucoup plus que tous les raisonnements. Avec la formule "Dieu le veut " l'Europe fut lancée sur l'Orient à l'époque des croisades. La formule " dictature du prolétariat" ruina la Russie. La formule "l'Allemagne paiera" a créé des gaspillages financiers dont le poids nous écrase. 
Les réformateurs n'influencent les âmes qu'à la condition d'avoir pour soutiens des formules mystiques chargées d'espérances.

La puissance des formules politiques populaires disparaît généralement avec leur réalisation. Après avoir fait plusieurs révolutions afin d'obtenir le suffrage universel, les révolutionnaires de divers pays fascistes en Italie, sinn-feiners en Irlande, communistes en Russie, syndicalistes en France, etc., le rejettent de plus en plus pour lui substituer des formes diverses de dictature.

Constituer un parti politique revient généralement à revêtir de noms nouveaux des choses fort anciennes.

Les réalités cachées sous les formules n'ont souvent aucun rapport avec ces formules. Lorsque, par exemple, un gouvernement réclame la liberté des détroits conduisant à Constantinople, cela signifie simplement qu'il voudrait devenir maître de ces détroits pour empêcher, au besoin, ses rivaux d'y pénétrer.

Sur mille hommes répétant avec enthousiasme une formule politique pour laquelle ils sont prêts à sacrifier leur vie, on n'en trouverait souvent aucun capable de définir exactement le sens de cette formule.

\section{V - Les erreurs de psychologie en politique}

$\underline{\text { Retour à la table des matières }}$

Le chaos où s'enlise l'Europe dérive tout autant d'une succession d'erreurs psychologiques que des perturbations économiques créées par la guerre.

La logique qui mène le monde étant sans rapport avec la logique livresque, il serait dangereux pour un pays d'avoir à sa tête trop d'hommes uniquement formés par les livres.

Dans les temps modernes, les erreurs politiques sont chargées de conséquences formidables. Les Anglais ont perdu l'Égypte, la Mésopotamie, la Perse et voient leur puissance menacée dans l'Inde pour avoir voulu rayer de l'Europe la Turquie, considérée par tous les musulmans comme le centre de leur foi. 
$\mathrm{Ne}$ pas tenir compte d'événements possibles, mais improbables, est toujours imprudent. L'intervention de l'Amérique, la trahison de la Russie, la défaite de l'Allemagne après de nombreuses victoires et bien d'autres événements de la dernière guerre montrent, une fois de plus, le rôle de l'improbable en histoire.

Dans un parlement ou une assemblée délibérante quelconque, le parti qui gouverne en réalité n'est pas le plus nombreux, mais le plus violent. Notre Parlement resta pendant vingt ans dominé par une minorité socialiste.

Malgré les illusions socialistes, le travail collectif exige des capacités d'autant plus hautes qu'il est plus collectif. C'est pourquoi notre époque a besoin de beaucoup plus de chefs qu'elle n'en trouve. Le célèbre industriel allemand, Hugo Stinnes, disait à ce sujet "Si cette décadence de l'individualité continue, aucun progrès n'est plus possible."

Pour ôter aux socialistes leurs illusions sur les avantages de l'administration étatiste, il leur suffirait de remarquer que dans certaines entreprises gérées par l'État, telles que les postes, les frais du personnel représentent $78 \%$ des dépenses totales de l'exploitation. Aucune industrie, aucun commerce ne pourrait vivre dans des conditions semblables.

Aux époques agitées, les grands problèmes qui surgissent chaque jour ne comportent guère de solutions simples et immédiates. Suivre alors l'opinion simpliste des foules conduit vite à des catastrophes.

Les impulsifs sont toujours dangereux, car les réalités échappent à l'homme qui agit sans réfléchir. Les êtres capables de réflexion, mais dépourvus de volonté, sont aussi nuisibles parce que leur irrésolution les paralyse devant les événements exigeant une décision immédiate.

En politique, les conséquences d'un acte ont parfois plus d'importance que cet acte lui-même.

Bien des catastrophes seront évitées le jour, probablement lointain, où les gouvernants posséderont un thermomètre psychologique capable de leur apprendre quand il faut résister et quand il faut céder. Charles Ier perdit sa tête pour avoir trop résisté; Louis XVI pour avoir trop cédé.

Une pénétration psychologique supérieure peut seule prévoir les réactions de l'âme des peuples sous des influences diverses. Les Allemands n'auraient pas irrité l'Amérique et perdu la guerre, si leurs chefs avaient possédé une telle pénétration. 
Une des plus fréquentes sources d'erreurs politiques est d'attribuer à des causes uniques des événements issus de causes nombreuses et compliquées.

C'est la série d'erreurs psychologiques commises par les Alliés qui permit à l'Allemagne d'obtenir ces deux grands résultats : dissocier l'Entente et rendre impossible par la dépréciation de sa monnaie le paiement de l'indemnité due aux vainqueurs.

La véritable force de l'Autriche résidait dans les aspirations contraires des races différentes qui la composaient. Ce grand empire était fondé sur un équilibre de haines.

Quelle que soit l'intelligence d'un homme d'État, en arrivant au pouvoir il cherche à suivre l'opinion mobile des foules pour se rendre populaire. C'est ainsi que, souvent, il perd le pouvoir.

La crainte des électeurs, la peur des responsabilités, la préoccupation exclusive de l'heure présente, constituent pour un homme politique moderne trois sources d'erreur auxquelles il lui est difficile d'échapper.

Les interventions étatistes troublant le jeu des lois naturelles sont chargées d'incidences invisibles qui perturbent profondément la vie d'un pays. La taxe sur le blé pendant la guerre en constitue un exemple frappant. Les paysans délaissèrent aussitôt sa culture et le gouvernement dut se procurer à grands frais, au dehors, le blé nécessaire, puis abolir la taxe.

Un gouvernement faible a pour terminaison nécessaire un gouvernement anarchique, auquel succède bientôt un gouvernement despotique.

L'impartialité en politique est impossible parce que l'homme impartial aurait immédiatement contre lui tous les partis, y compris celui auquel il appartient.

Dans le régime démocratique, les chefs sont souvent plus disposés à obéir qu'à commander. Ils finissent ainsi par perdre tout prestige.

Suivre toujours l'opinion mobile des multitudes, c'est se résigner à ne rien prévoir, rien empêcher, rien pouvoir.

En politique comme en agriculture, on récolte ce qu'on a semé. Les Anglais, reprochant à la France d'avoir favorisé la Turquie, oubliaient les ennuis que pendant quatre ans ils nous suscitèrent un peu partout. 
Les erreurs politiques peuvent engendrer par contagion mentale des épidémies dévastatrices. La contagion bolcheviste a fait périr plus d'hommes que bien des batailles et ramené la Russie aux périodes sauvages de la préhistoire. eux.

L'homme d'État qui ne sait pas orienter les événements est bientôt submergé par

Bien que la politique soit certainement l'art dont la pratique exigerait le plus de jugement, c'est celui où il s'en dépense le moins.

L'arsenal psychologique contient des armes qui, bien maniées, peuvent dépasser le pouvoir des canons. Ce maniement, que n'enseignent pas les livres, exige une longue expérience. 
Les incertitudes de l'heure présente

\section{Chapitre II}

\section{Les guerres, les révolutions et le désarmement}

\section{I - Les causes futures de guerre et la revanche germanique}

$\underline{\text { Retour à la table des matières }}$

Les anciennes guerres, dérivant surtout de l'ambition personnelle des souverains, n'avaient aucun caractère de nécessité. Les conflits modernes résultant de luttes d'intérêts collectifs sont bien plus difficilement évitables. Alexandre et César pouvaient ne pas entreprendre leurs conquêtes. De nos jours, la volonté de l'empereur d'Allemagne fût, tôt ou tard, devenue impuissante à dominer les aspirations d'un peuple hanté par le besoin mystique d'hégémonie.

Les accroissements de territoires récemment réalises par l'Angleterre montrent à quel point l'idée de s'agrandir au moyen de conquêtes reste une des conceptions directrices de certains peuples. 
À l'heure de la victoire, il est facile au vainqueur d'imposer ses volontés. Cette possibilité s'atténue progressivement jusqu'au jour où la résistance du vaincu ne pouvant plus être anéantie que par la force, une nouvelle guerre devient nécessaire.

Les peuples ne se résignent pas à la défaite quand ils se croient supérieurs à leurs vainqueurs. Une tentative de revanche germanique peut donc être considérée comme un des plus sûrs événements de la future Histoire.

L'idéal de l'empereur Guillaume, d'après ses Mémoires, était d'avoir une armée et une flotte assez puissantes pour que nul n'osât les attaquer. Il oubliait alors que le possesseur de pareils moyens de défense songe bientôt à les utiliser pour se débarrasser de rivaux gênants. Ce fut justement la notion de sa force qui le conduisit à la guerre.

Deux formes de revanche sont rêvées en Allemagne : $1^{\circ}$ par les armes; $2^{\circ}$ par l'expansion commerciale. Le succès de la seconde tentative entraînerait fatalement la réalisation de la première.

L'unique moyen - en dehors de formidables armements — d'empêcher une future agression de l'Allemagne, eût été son retour aux provinces autonomes qui la composaient avant 1871. Elle-même le réclamait après l'armistice, pour se soustraire à la domination prussienne. Les historiens s'étonneront sûrement que les auteurs du traité de paix n'aient pas compris une telle évidence.

Beaucoup de phénomènes sociaux possèdent un point critique comparable à celui de certains phénomènes physiques. Dans son voisinage, de faibles influences peuvent déterminer des changements très grands, la paix ou la guerre, par exemple. L'origine des guerres de 1870 et de 1914 vérifie cette observation.

Le conflit mondial a révélé deux principes que les guerres antérieures ne permettaient pas de pressentir. Le premier, que le vainqueur se trouve aussi ruiné que le vaincu. Le second, que les indemnités incombant au vaincu sont indirectement payées par les autres peuples, y compris ceux qui n'ont pris aucune part au conflit.

Le capital matériel d'un peuple peut être détruit dans une guerre. Le capital moral, constitué par l'intelligence, le pouvoir d'organisation et la capacité technique, étant indestructible, permet de reconstituer rapidement le capital matériel. L'Allemagne en fournit un nouvel exemple.

La prochaine histoire de l'Europe dépendra surtout de l'intérêt qu'auront les grandes nations à prolonger, durant la paix, les alliances formées pendant la guerre. 
Il est de toute évidence que si trois grands pays comme la France, l'Angleterre et l'Amérique avaient accepté de s'unir contre un agresseur quelconque, la paix se fût trouvée assurée. Les divergences d'intérêts et de mentalité de ces nations et la méfiance réciproque de leurs gouvernants, ayant empêché cette alliance de se réaliser, les peuples sont condamnés à se ruiner en armements.

Si la raison pouvait exercer un rôle quelconque sur les relations entre les peuples, ils seraient vite persuadés que leur intérêt est de s'entr'aider au lieu de s'entredétruire.

\section{II - Les luttes pour l'hégémonie}

$\underline{\text { Retour à la table des matières }}$

Les conséquences réelles de la grande guerre diffèrent beaucoup des résultats prévus. Certains peuples s'aperçoivent maintenant qu'après avoir écarté au prix de gigantesques efforts l'hégémonie militaire allemande, il leur faut subir l'hégémonie économique et politique de l'Angleterre. Elle ne parait pas moins lourde.

L'hégémonie anglaise n'a plus aujourd'hui d'autre contrepoids que le pouvoir grandissant des États-Unis.

Parmi les signes divers révélant l'aspiration à l'hégémonie figure le langage des diplomates. L'Angleterre s'est emparée des flottes et des colonies germaniques, puis a proclamé son protectorat sur la Perse et l'Égypte ; mais quand les alliés voulurent défendre leurs droits, le langage des dirigeants anglais devint agressif. Le monde comprit alors qu'une hégémonie nouvelle était née.

Le jour où l'expérience prouva que, malgré flottes et sous-marins, l'Amérique pouvait envoyer en Europe un million d'hommes armés, la situation mondiale de l'Angleterre s'est trouvée virtuellement transformée. La domination des mers et la suprématie commerciale lui échapperont fatalement dans l'avenir au profit de l’Amérique.

Si l'Angleterre avait réussi, au moyen de ses tentatives répétées, à empêcher la France d'exiger les réparations dues par l'Allemagne, elle eût retiré de la guerre ces deux immenses résultats : $1^{\circ}$ se débarrasser de la rivalité maritime allemande $; 2^{\circ}$ supprimer la rivalité commerciale possible de la France, forcée de consacrer toutes ses ressources à la restauration des départements dévastés. 
Lorsque l'Allemagne rêvait d'une paix universelle par l'établissement de son hégémonie, elle subissait une illusion psychologique dont furent victimes tous les grands conquérants. Son succès eût fatalement déclenché une série de coalitions qui auraient détruit sa puissance, comme le fut celle de Napoléon.

La grande rivalité entre l'Angleterre et l'Allemagne s'est terminée par l'hégémonie anglaise en Europe. La lutte pour l'hégémonie de l'Asie ne fait que commencer.

L'importance donnée au Japon par la guerre et sa rapide conquête du Pacifique hâteront nécessairement le choc colossal entre la race blanche et la race jaune. Les résultats du conflit diront dans quelles mains le sceptre de l'Asie devra passer.

Les hommes d'État actuels se défendent bien haut de toute pensée d'impérialisme et affectent de considérer cette accusation comme une injure. Ils savent cependant que seules des visées impérialistes ont édifié et fait prospérer les grands empires dont est formée l'Europe.

Le premier acte de la guerre mondiale, la lutte militaire, est aujourd'hui terminé. Le second acte, la guerre économique, commence. Le troisième acte, lutte de la race jaune contre la race blanche pour l'hégémonie de l'Asie, semble prochain.

Si la notion d'interdépendance des peuples n'arrive pas à remplacer celle d'hégémonie, l'Europe devra subir des guerres d'extermination qui la plongeront dans une décadence sans espoir.

\section{III - Les illusions sur la possibilité d'un désarmement}

$\underline{\text { Retour à la table des matières }}$

A tous les âges de l'histoire, et aujourd'hui plus que jamais, le respect qu'inspire un peuple dépend surtout de son prestige militaire.

Les grandes nations modernes sont oppressées par ce dilemme renoncer à leurs armements pour éviter une ruine financière, ou les accroître pour empêcher des invasions plus coûteuses encore. 
Un peuple ne se protège contre les attaques de ses rivaux qu'en restant fort. Si les idées pacifistes propagées avant la guerre par les socialistes n'avaient pas considérablement affaibli notre préparation militaire, il est infiniment probable que l'Allemagne n'aurait jamais songé à nous attaquer.

Guillaume II, dans ses Mémoires, écrit un véritable traité de la guerre et de la paix en deux lignes quand il dit qu'un peuple doit être assez armé sur terre et sur mer pour créer chez son adversaire la peur du risque. On ne se permet guère, en effet, d'attaquer les forts, alors que les faibles restent toujours menacés.

Les guerres ne pourront disparaître qu'avec la suppression des causes qui les font naître : haine entre les races, besoin d'hégémonie des peuples forts, rivalités économiques, etc. La science devra donc d'abord découvrir un moyen de transformer complètement la nature de l'homme.

Depuis les origines de l'histoire, les relations entre peuples faibles et peuples forts furent exactement celles du gibier avec le chasseur.

L'idée finit quelquefois par dominer le canon, mais privée de la protection du canon elle reste sans force.

Les philosophes soutenant que la philanthropie occasionnera encore plus de ravages que les épidémies et les canons auraient peut-être raison, si les harangues des philanthropes avaient jamais exercé une influence quelconque sur la conduite des peuples. Mais elles ne servent guère qu'à orner des discours.

Aimez-vous les uns les autres, conseillent inutilement les religions; supportezvous, exigent simplement les codes. Aidez-vous deviendra la maxime de l'avenir quand les peuples auront découvert l'interdépendance qui les lie.

Dans l'état de déséquilibre du monde actuel, le terme désarmement est synonyme de servitude. 


\section{IV - Les incertitudes sur les origines de la guerre}

$\underline{\text { Retour à la table des matières }}$

Il n'est pas toujours facile de découvrir les vrais auteurs d'une guerre. Il fallut longtemps pour reconnaître qu'en 1870, le roi de Prusse et l'Empereur des Français firent la guerre malgré eux.

Les origines de la guerre mondiale sont incompréhensibles quand on l'imagine issue de la volonté des trois empereurs qui l'ordonnèrent. Il faut l'envisager comme la résultante inévitable non seulement de l'histoire des États européens depuis un siècle, mais aussi de l'enseignement des historiens et des universités germaniques depuis cinquante ans.

Pour écrire aujourd'hui l'histoire de la grande guerre et de ses causes, ce ne sont pas les documents qui manquent mais la sérénité de jugement permettant de l'étudier comme s'il s'agissait d'événements anciens, tels que les guerres puniques ou la bataille d'Actium.

Il est probable qu'en 1914 l'empereur d'Allemagne n'eût pas déclaré la guerre si l'Angleterre avait manifesté plus tôt son intention de s'unir à la France, mais étant donné l'état d'esprit créé par les militaires et les universitaires allemands, il semble que le conflit eût été simplement retardé.

Les guerres franco-allemandes ne sont intelligibles que considérées comme des phases successives d'un conflit séculaire dont la fin ne s'entrevoit pas encore.

Quand on recherche les origines lointaines de la dernière guerre, il apparaît vite qu'une lutte armée entre l'hégémonie allemande et l'hégémonie anglaise était aussi fatale que le fut, jadis, celle entre Rome et Carthage. 


\section{$\mathrm{V}$ - Les causes des révolutions}

$\underline{\text { Retour à la table des matières }}$

Les peuples vivent surtout d'espérances. Leurs révolutions ont pour but de substituer des espérances nouvelles à d'anciennes espérances devenues sans force.

Un pays est voué aux révolutions dès que les partis ayant intérêt à défendre l'ordre établi deviennent moins énergiques que ceux qui aspirent à le détruire.

Le principal résultat des révolutions qui bouleversent l'histoire est de changer les chefs incarnant le principe d'autorité. Les multitudes profitent rarement de cette substitution.

Lorsque les nécessités économiques sont contraires aux impulsions affectives et mystiques qui mènent les hommes, une révolution devient inévitable.

L'envie et son inséparable compagne, la haine, forment les grands ressorts des révolutions sociales. La Révolution Française eut pour cause initiale les différences extérieures et les privilèges qui séparaient la bourgeoisie de la noblesse. La révolution sociale dont nous sommes menacés comptera parmi ses origines les distinctions existant, non dans les codes, mais dans les mœurs, entre les diverses classes.

Quand la haine remplace chez l'inférieur le respect du supérieur, une révolution est proche.

Le bolchevisme représente un état mental qui a plusieurs fois sévi dans l'histoire. Ses éléments psychologiques furent toujours les mêmes indiscipline, haine jalouse des supériorités, désir intense de s'emparer par la violence des biens qu'on se sent incapable d'acquérir par le travail ou l'intelligence.

Les civilisations modernes traînent derrière elles une foule croissante d'inadaptés rêvant de les détruire afin de leur substituer des formes de vie sociale moins compliquées, telles que le communisme.

L'armée des inadaptés devient aussi menaçante aujourd'hui que les futures invasions germaniques. 
Un des grands problèmes modernes consiste à savoir si des bolchevistes sans culture - ou, ce qui est plus dangereux encore, munis d'une demi-culture, — réussiront à ramener nos grandes civilisations à des types inférieurs voisins de la barbarie.

La mentalité d'un peuple déterminant rigoureusement ses institutions et ses lois, certaines nations l'Irlande en Europe, les républiques latines dans l'Amérique, semblent condamnées par leur âme même à ne jamais sortir des révolutions et de l'anarchie.

La Révolution Française, faite par la bourgeoisie contre la noblesse, réussit parce que les capacités de cette bourgeoisie étaient devenues supérieures à celles de la noblesse. De nos jours, une révolution prolétarienne pourrait momentanément triompher, grâce à la puissance du nombre, mais elle ne saurait durer parce que l'évolution industrielle exige des compétences directrices que l'ouvrier ne possède pas.

Un parti révolutionnaire doué de prestige rallie facilement des mécontents de tous les autres partis. Les bolchevistes recrutent des adeptes en Perse et en Turquie, bien que l'islamisme et le communisme n'aient aucun caractère commun.

\section{VI - Les résultats des révolutions}

$\underline{\text { Retour à la table des matières }}$

La première phase d'une révolution est consacrée à combattre les nécessités économiques et sociales qui régissent la vie des peuples. L'expérience prouvant bientôt que ces nécessités dominent les volontés, l'ancienne organisation reparaît sous des noms nouveaux. Ainsi se terminera nécessairement la révolution russe.

Les rêveurs n'ont aucun pouvoir créateur, mais ils possèdent parfois une puissance destructive considérable. Sous leur dissolvante action, les institutions péniblement édifiées par le temps se désagrègent avec une extrême rapidité. Quelques mois suffirent aux communistes russes pour ramener leur pays à la barbarie.

L'imagination créatrice prépare l'invention. Le laboratoire en fixe les contours. L'usine la transforme en éléments de progrès qu'utilisent tous les hommes. Le massacre des intellectuels par les communistes russes montre combien est ignorée des foules cette genèse des découvertes dont elles profitent. Le bolchevisme a révélé le 
degré de misère auquel peut tomber l'ouvrier privé de la pensée capable d'orienter ses efforts.

Si équitable que puisse être un idéal révolutionnaire, il ne triomphe qu'au prix de guerres acharnées. Vingt années de luttes meurtrières furent nécessaires pour établir en Europe le principe d'égalité devant la loi et supprimer les privilèges de certaines classes.

Un gouvernement révolutionnaire ne subsiste qu'à la condition de tomber sous le despotisme de quelques meneurs.

Les révolutions ne durent jamais longtemps parce qu'elles se heurtent bientôt au mur des nécessités économiques et sociales qui dominent le monde. Percevant alors l'impuissance des théoriciens, la foule se détourne d'eux. Avant d'arriver à cette dernière phase, bien des ruines sont accumulées. La Russie en fait aujourd'hui l'expérience.

Les révolutions enrichissent quelques-uns des chefs qui leur survivent, mais elles augmentent invariablement la misère des foules qui les ont réalisées. Cette vérité étant inaccessible aux multitudes, les meneurs révolutionnaires pourront continuer longtemps à bouleverser le monde.

L'histoire des assemblées révolutionnaires de tous les temps montre que les fanatiques n'ont encore découvert aucune autre méthode de persuasion que le massacre systématique de leurs adversaires.

Ce n'est pas à la liberté mais à la servitude que beaucoup de révolutionnaires modernes aspirent sans le savoir. La liberté n'est conçue par eux que sous forme de soumission à un maître dont les moindres paroles sont des oracles. Toutes les révolutions modernes se terminent par la création d'un autocrate.

La soif d'inégalité semble un besoin irréductible de la nature humaine. On sait avec quelle ardeur les Conventionnels échappés à la guillotine sollicitaient de Napoléon des titres nobiliaires. Le rêve égalitaire qui les avaient conduits à tant de massacres n'était donc en réalité qu'un violent désir d'inégalité à leur profit. L'histoire n'a pas encore cité, d'ailleurs, de pays où régnât l'égalité.

Jamais baron féodal ne manifesta pour les serfs un mépris égal à celui que témoignent au peuple les chefs des nouveaux partis révolutionnaires, le communisme russe notamment. Dès l'arrivée au pouvoir des dictateurs bolchevistes, la liberté de la presse, la journée de huit heures, le suffrage universel, furent supprimés et l'ouvrier devint un simple esclave. 
On ne rencontre guère d'exemple dans l'Histoire de révolutions n'ayant pas finalement engendré des résultats absolument contraires à ceux que poursuivaient leurs auteurs.

La Révolution bolcheviste est une de celles qui montrent le mieux combien les buts atteints par les révolutions peuvent différer des buts poursuivis. Elle triompha en promettant la paix, et se trouva bientôt en guerre avec tous ses voisins. Elle voulait supprimer le militarisme et n'a fait qu'établir un régime militaire plus dur que tous les régimes antérieurs. Elle prétendait abolir le droit de propriété et n'a réussi qu'à créer la propriété individuelle dans un pays qui n'avait encore connu que la propriété collective.

Étant donné le nombre immense de paysans russes devenus propriétaires et possédant, dès lors, la mentalité particulière que détermine la propriété, on peut affirmer que la Russie sera bientôt le pays du monde qui renfermera le moins de socialistes.

Ce n'est pas d'une révolution, mais d'une transformation profonde des idées que résultent les réformes durables.

D'après tous les enseignements de l'histoire des révolutions, l'extrémisme en politique a comme terminaison nécessaire soit la destruction de la civilisation où il sévit, soit l'anarchie et la dictature.

]1 faut beaucoup d'années à un peuple pour acquérir un équilibre durable et peu de temps pour le perdre. 
Les incertitudes de l'heure présente

\section{Chapitre III}

\section{Les relations internationales et les alliances}

\section{I - Les relations internationales}

$\underline{\text { Retour à la table des matières }}$

Alors que la solidarité des peuples devrait être la loi des temps modernes, une haine intense plane sur l'univers. Haine entre nations, entre classes diverses d'une même nation, haine entre partis politiques de chaque classe. L'interdépendance des peuples est une nécessité qui finira peut-être par les solidariser, mais son influence reste actuellement nulle.

La haine entre peuples, la défiance entre gouvernants, sont devenues les grands ressorts psychologiques de la politique actuelle. On put évaluer l'intensité de ces sentiments quand on vit l'Amérique et l'Angleterre renoncer à la paix future, que leur promesse d'intervention en cas d'attaque de l'Allemagne eût rendue certaine.

$\mathrm{Si}$, dans leurs relations, les individus se conduisaient avec autant de mauvaise foi et de méfiance que les peuples entre eux, aucune société ne pourrait durer. 
L'union entre citoyens à l'intérieur, entre alliés à l'extérieur, est reconnue comme seule base possible d'une paix durable. Les haines grandissantes de peuple à peuple et d'une classe à l'autre, dans un même pays, prouvent malheureusement que des intérêts évidents, mais lointains, restent sans force contre les impulsions passionnelles du moment.

L'amitié entre individus peut subsister malgré les différences d'intérêts. Entre peuples, l'amitié ne représentant qu'une communauté d'intérêts ne saurait survivre à leurs divergences. Ce principe de psychologie collective conditionne la durée possible d'une alliance.

Le monde oscille aujourd'hui entre le nationalisme impérialiste et l'internationalisme. Le nationalisme implique, avec la solidarité sociale, le culte de la patrie. L'internationalisme, remplaçant la solidarité par la lutte des classes, rendrait un peuple aussi impuissant à se protéger contre les guerres civiles que contre les invasions.

Le nationalisme, qui seul donne aux peuples leur cohésion, a des chances de durée parce qu'il constitue un sentiment naturel. Mais la force même de ce sentiment le fait souvent dégénérer en impérialisme agressif. Il substitue alors les guerres extérieures aux guerres intérieures.

Si intense soit la haine entre peuples, elle n'est jamais aussi vive qu'entre les partis politiques d'un même peuple.

Le monde changera de face et les rapports entre les divers pays seront profondément transformés lorsque les politiciens qui en dirigent les destinées auront découvert que l'intérêt d'une nation n'est pas basé sur le dommage causé à une autre. Ils renonceront alors au besoin d'hégémonie qui continue d'aveugler l'esprit des gouvernants.

En politique internationale, les coups d'épingle répétés finissent par engendrer des coups de canon.

\section{II - Les forces économiques}

$\underline{\text { Retour à la table des matières }}$

Ce n'est plus la volonté des dieux, mais les lois économiques qui, dans les temps modernes, déterminent la destinée des nations.

Si un bouleversement géologique avait détruit, il y a un siècle, toutes les mines de charbon et de pétrole de l'univers, cet événement n'aurait entraîné aucune conséquence importante. S'il se produisait aujourd'hui, les chemins de fer et les usines ces- 
seraient aussitôt de fonctionner. La régression immédiate de nos civilisations en résulterait. Un abîme sépare le monde actuel de son état antérieur.

Les discours, les conférences, les lois elles-mêmes sont impuissantes à combattre les nécessités économiques qui étreignent le monde. Il faut s'y adapter ou périr.

Les vérités scientifiques s'établissent facilement parce qu'elles s'adressent à l'intelligence. Les vérités économiques, se heurtant à des sentiments et à des illusions sociales, ne s'imposent qu'après de désastreuses expériences. La ruine de la Russie en est le plus récent exemple.

Dans les questions économiques et sociales, les intérêts déterminent l'opinion et les institutions qui en dérivent. Les Anglais resteront libre-échangistes, les Américains protectionnistes, tant qu'ils trouveront un avantage à maintenir leurs doctrines.

Dans une guerre prolongée, il arrive un moment où vaincu et vainqueur étant également ruinés, les indemnités exigibles du vaincu ne peuvent jamais compenser les pertes subies par le vainqueur.

On a justement observé que ce sont les pays à change élevé qui souffrent le plus du chômage.

Le change, en effet, a pour les acheteurs exactement les mêmes conséquences qu'une élévation considérable du prix des marchandises. Ces marchandises devenant alors presque invendables, il en résulte la fermeture des usines et le chômage.

Un peuple vivant d'emprunts étrangers tombe fatalement sous la dépendance du prêteur obligé de surveiller les garanties de sa créance. Une nation assez riche pour prêter beaucoup à une nation pauvre finirait par la dominer plus étroitement qu'au moyen d'une conquête militaire.

La richesse d'un pays ne réside pas dans des billets sans garantie qu'il peut émettre à volonté, mais dans son industrie et son agriculture. L'Allemagne, n'ayant perdu ni ses champs ni ses usines, reste presque aussi riche qu'avant la guerre malgré la perte à peu près totale de sa monnaie.

Les problèmes financiers actuels n'auront de solution possible qu'étudiés en fonction du temps. Avec son concours, la dette la plus colossale devient aussi petite qu'on le désire. La somme la plus minime, au contraire, peut devenir considérable. Les collectivités seules sont capables de réaliser des combinaisons basées sur la puissance du temps, parce que leur vie est illimitée. 
Le change représente simplement le degré de la confiance du monde dans le crédit d'un État. On ne stabilise pas plus le change qu'on ne stabilise un baromètre ou tout autre instrument de mesure. Les oscillations du change révèlent surtout les oscillations de la confiance.

Une grève pour l'augmentation des salaires ne représente pas en réalité une lutte entre ouvriers et patrons, mais entre les ouvriers et le public. C'est le public, en effet, qui paie toujours l'élévation de prix déterminée par le succès d'une grève. S'il prend généralement le parti des grévistes, c'est que les collectivités sont incapables de saisir les conséquences un peu lointaines des phénomènes.

Les énergies invisibles qui mènent le monde sont comparables à l'électricité, force de nature ignorée, connue seulement par les résultats visibles qu'elle produit.

\section{III - Les traités de paix et les congrès politiques}

$\underline{\text { Retour à la table des matières }}$

L'idée que des collectivités puissent découvrir la solution de problèmes ayant échappé à des individualités compétentes serait reconnue erronée depuis longtemps si les déterminations collectives ne représentaient, le plus souvent, la décision d'une personnalité assez forte pour s'imposer. La résolution supposée collective n'est alors, en réalité, qu'une décision personnelle.

Il y a peu de rapports entre les principes formulés par les hommes d'État dans les congrès et ceux qui guident leur conduite. Pendant les conférences de la paix, l'âme des diplomates anglais fut dominée par trois principes inavoués : $1^{\circ}$ accroître les possessions britanniques, $2^{\circ}$ empêcher la France de devenir trop forte, $3^{\circ}$ laisser l'Allemagne assez puissante pour faire équilibre à la France.

Il faudrait supposer beaucoup de candeur aux dirigeants britanniques pour admettre qu'ils aient cru remédier à la situation économique de l'Europe en réunissant des conférences. Le but poursuivi était donc tout autre que le but proclamé.

La plupart des congrès de l'histoire ont fait surgir des causes de guerre et n'en ont empêché aucune. 
La seule utilité possible d'un congrès aux yeux des hommes politiques assez influents pour imposer leur volonté, est de renforcer par l'autorité du nombre les décisions personnelles qu'ils veulent faire accepter.

Si la rédaction du traité de paix eût été soumise à l'opinion publique, et non discutée en secret, toute la subtilité de certains diplomates n'aurait jamais fait admettre que le chiffre des réparations dues à la France serait fixé par une commission composée en majorité des représentants de pays n'ayant aucun intérêt à ces réparations.

Les conséquences du traité de paix qui termina la grande guerre, montrent combien fut complète la cécité mentale de ses auteurs, expulsant la Turquie de l'Europe et découpant l'Autriche en petits États sans ressources économiques pouvant les soustraire à la misère et à l'anarchie. Il devient évident aujourd'hui que, dans l'intérêt de l'Europe, l'unité de l'Autriche devait être soigneusement conservée et l'Allemagne ramenée à des États séparés comme elle l'était avant de tomber sous la domination prussienne.

La rédaction du traité de Versailles ne fut pas gênée seulement par la contradiction des intérêts en présence, mais aussi par le désir de céder à certaines exigences en raison de la reconnaissance due à l'Angleterre et à l'Amérique. Venus, croyait-on, au secours de la France, pour sauvegarder le droit et la justice, ces États reconnaissent aujourd'hui être entrés dans la lutte uniquement pour défendre leurs intérêts menacés.

Les conférences atteignent fréquemment un but contraire à celui qu'elles se proposaient. La conférence de Gênes ne fit que consolider la situation des soviets et favoriser le rapprochement, si opposé aux intérêts français, de la Russie et de l'Allemagne.

Lorsque des diplomates se réunissent pour résoudre une question sur laquelle ils sont certains de ne pas s'entendre, la discussion se porte immédiatement sur des détails accessoires afin d'éviter le sujet principal. C'est ainsi qu'à Washington la question du libre établissement des Japonais aux États-Unis, qui obsédait tous les esprits, ne fut même pas effleurée.

\section{IV - Les traités d'alliance et leur valeur}

$\underline{\text { Retour à la table des matières }}$

Il devient impossible de prévoir où conduira une alliance, et c'est sans doute pourquoi certains grands peuples hésitent aujourd'hui à en contracter. Ils n'ont pas oublié que l'alliance de la France avec la Russie entraîna une guerre ruineuse, et que la trahison de l'alliée pour laquelle nous étions entrés en lutte avec l'Allemagne faillit nous faire perdre la guerre. 
Quand on étudiera les origines de la grande guerre il faudra remonter assez loin pour déterminer la genèse des sentiments qui animaient alors les divers pays. Si, par exemple, la Russie se détourna de l'Allemagne pour aller vers la France, ce fut surtout parce que Bismarck, après le conflit russo-turc, empêcha les Russes de prendre Constantinople.

L'empereur Guillaume le rappelle dans ses Mémoires lorsqu'il dit qu'en 1914 " la revanche pour Sedan s'unit à la revanche pour Stefano ".

Un allié trop puissant est parfois aussi redoutable qu'un ennemi déclaré. L'alliance d'un peuple faible avec un peuple fort ne constitue généralement pour le peuple faible qu'une forme atténuée de la servitude.

L'alliance de plusieurs peuples durant une guerre est généralement très stable, parce que leurs intérêts sont alors identiques. L'union pendant la paix s'affaiblit au contraire très vite parce que les intérêts en présence deviennent bientôt divergents.

Les traités d'alliance ou de paix perpétuelle ne peuvent jamais être une oeuvre d'entière bonne foi. Les politiciens qui les signent connaissent trop bien l'Histoire pour ignorer qu'un pacte entre puissances liées par des intérêts communs se dissout dès que disparaît la communauté d'intérêts qui le fit naître.

Parmi les difficultés qui accompagnèrent la rédaction du traité de paix figura l'obligation où se trouvaient nos gouvernants de choisir entre une solitude pleine de futurs dangers et une alliance fertile en déboires, mais qui semblait nécessaire. Il n'est pas certain que la solution choisie fut la meilleure.

Une entente sans écrit vaut mieux, dit-on, qu'un écrit sans entente. Il ne faut pas oublier, cependant, que ce fut surtout l'absence d'écrit entre la France et l'Angleterre qui fit espérer à l'Allemagne la neutralité britannique, et lui suggéra de nous déclarer la guerre.

Les traités d'alliance chargés de réticences sont plus dangereux qu'utiles par la fausse sécurité qu'ils

inspirent.

Lorsque, après avoir été un lien qui unit, les alliances deviennent une chaîne qui entrave, leur désagrégation est prochaine. 


\section{V - La société des nations}

$\underline{\text { Retour à la table des matières }}$

Le palais de la Société des nations à Gênes est aux yeux des psychologues un palais d'illusions, mais ces illusions se trouvent enveloppées d'espérances assez fortes pour en voiler la médiocrité. Les peuples s'imaginent que cet aréopage de sages vieillards découvrira le moyen de soustraire le monde à l'enfer de ruines et de désolation auquel aboutissent les guerres modernes.

Les juristes vertueux mais bornés qui, aux conférences de la Haye, prétendaient proscrire l'usage de certaines armes, ne soupçonnaient pas la supériorité militaire qu'ils auraient ainsi attribuée aux pays dédaignant leurs prescriptions sur ceux qui les auraient respectées.

Les rêveries humanitaires, et notamment Je principe des nationalités, ont contribué à plonger l'Europe dans un état d'anarchie dont nul ne peut prévoir le terme.

La Société des nations restera une absurdité psychologique jusqu'au jour où elle possédera une force morale ou matérielle suffisante pour la transformer en un superÉtat dont les décisions seraient aussi universellement respectées que celles des Papes au moyen âge.

Tous les projets d'arbitrage international par une Société des nations semblent devoir rester bien illusoires lorsqu'il s'agira de concilier des intérêts nettement inconciliables. Était-il un arbitrage possible entre Rome et Carthage à l'époque des guerres puniques ou entre l'Angleterre et la France quand elles se disputaient la domination de l'Inde ? Nul arbitrage n'est possible entre le Japon cherchant à déverser sur les États-Unis l'excédent de sa population et la grande République qui refuse l'invasion de la race jaune. De tels intérêts étant aussi différents que ceux du mouton et du boucher, du gibier et du chasseur, la guerre constitue le seul arbitre dont les décisions soient respectées.

La lutte récente des Turcs contre les Grecs montre une fois de plus que certaines questions ne peuvent être résolues que par le canon. 
Les incertitudes de l'heure présente

\section{Chapitre IV}

\section{Le droit et la morale}

\section{I - Les coutumes et les lois}

$\underline{\text { Retour à la table des matières }}$

Le roi, la loi et l'opinion représentent les divers principes de gouvernement. Les rois ayant perdu leur prestige restent sans force. L'opinion est trop mobile pour donner de la stabilité à un peuple. La loi seule peut aujourd'hui créer la stabilité. Dès qu'elle cesse d'être respectée, l'anarchie commence.

Refuser d'obéir à un chef, à une loi, à une croyance, en un mot à une contrainte, c'est se condamner à n'avoir pour guides que des impulsions instinctives et retourner, par conséquent, à l'état de barbarie dont les peuples mirent tant de siècles à sortir.

La vie sociale et la vie scientifique constituent deux faces de la civilisation régies par des principes bien différents. Dans la vie sociale, le respect du principe d'autorité - autorité du chef, de la loi, des coutumes - est une condition fondamentale d'existence. Dans la vie scientifique, le rejet absolu du principe d'autorité représente, au 
contraire, la condition nécessaire du progrès. Dès que le principe d'autorité s'introduit dans une science, le développement de cette science s'arrête.

Édicter des lois violant les habitudes et les intérêts généraux, et ne pouvant donc être observées, c'est ébranler dans les âmes le respect des codes, ciment essentiel des grandes civilisations.

Prétendre combattre avec des lois les nécessités économiques qui mènent le monde est une dangereuse erreur. Les lois restrictives accumulées depuis quelques années pour obéir aux exigences de théoriciens simplistes, n'ont fait que paralyser la vie industrielle, agricole, économique de plusieurs peuples.

Les luttes de l'avenir entre les diverses classes d'un même peuple ne se feront probablement pas toujours à main armée. Elles se traduiront surtout, comme chez les anciens Grecs, par des lois sociales provoquant la ruine totale des plus faibles.

Les lois répressives deviennent préventives dès qu'elles sont imposées avec rigueur. La crainte du châtiment est alors plus efficace que son application. La méconnaissance de ce principe psychologique contribua beaucoup à l'accroissement de la criminalité dans divers pays.

Les effets d'une loi dépendent toujours de la mentalité des hommes qu'elle est destinée à régir. Les juristes répètent que les lois ne sont rien sans les mœurs, mais dès qu'ils se mettent à légiférer, ils oublient cette maxime.

Les lois sociales, qui représentent des contraintes artificielles, restent bientôt sans force. Les lois économiques résultant de nécessités naturelles s'imposent au contraire toujours, malgré les efforts tentés pour les violer.

Une des erreurs démocratiques les plus répandues est de croire que les lois peuvent établir des coutumes. En réalité, les coutumes engendrent finalement des lois, mais les lois ne créent que rarement des coutumes.

Parmi les milliers d'hommes aspirant à établir le règne du droit et de la justice, bien peu seraient capables de définir le droit et la justice. 


\section{II - Le droit et la force}

$\underline{\text { Retour à la table des matières }}$

La force ne prime pas le droit, mais le droit ne se démontre que par la force.

Le droit sans force est comparable aux décors de forteresses peints sur les toiles d'un théâtre. Incapables de résister au moindre choc, ils ne conservent leur aspect redoutable que si l'on n'y touche pas.

Une force quelconque ne peut évidemment être annulée que par une autre au moins égale. Assurer que le droit domine la force serait absurde si l'on ne sousentendait ainsi que le droit arrive à susciter des forces supérieures aux puissances matérielles qu'il doit vaincre.

On peut médire de la force et assurer qu'elle ne saurait triompher du droit, mais en politique rien ne la remplace. Quelques semaines avant la foudroyante victoire de Kemal, le premier ministre anglais dédaignait de recevoir son envoyé. Le lendemain de la victoire, le même ministre traitait sur un pied d'égalité avec la Turquie si méprisée la veille et lui abandonnait des provinces où avait flotté le drapeau britannique.

Le droit et la force acquièrent un grand pouvoir par leur association. La force seule n'engendre pas de succès durable. Les Allemands en ont fait l'expérience.

La force a toujours gouverné le monde, mais ce ne furent pas les mêmes forces qui prédominèrent aux divers âges de l'histoire. Les forces économiques tendent à devenir aussi souveraines du monde actuel que les forces religieuses le furent jadis.

Où sévit l'inégalité, sévit aussi l'injustice. Ne pouvant empêcher l'inégalité, loi irréductible de la nature, il faut bien se résigner à subir l'injustice.

Il est difficile de reprocher aux Allemands leur persistance à proclamer la supériorité de la force. Vaincus par les armées que leur besoin d'hégémonie déchaîna, ils voient à quel état misérable le manque de force peut conduire un peuple. 


\section{III - Les forces morales}

$\underline{\text { Retour à la table des matières }}$

Il n'est pas d'exemple de peuples arrivés à la civilisation sans discipline, sans respect des lois et sans morale.

Un peuple ne sort de la barbarie que par l'acquisition d'une morale très stable. Dès qu'il l'a perdue, il retourne à la barbarie.

La guerre a montré une fois encore que la puissance d'une nation réside beaucoup plus dans sa force morale que dans sa culture intellectuelle.

La lutte mondiale fut peut-être la première au cours de l'histoire où le succès final dépendit autant de la résistance des soldats que de la capacité des généraux. Ludendorff, dans ses.Mémoires, reconnaît que la guerre lui sembla perdue quand il vit fléchir le moral de son armée.

Les liens moraux peuvent devenir aussi forts que des liens matériels. Sur la trirème qui le ramenait volontairement à Carthage, où il se savait condamné à périr, le consul Régulus était attaché par sa parole plus rigoureusement qu'il ne l'eût été par des chaînes de fer. Rome domina le monde tant qu'elle posséda de tels hommes.

Les lois scientifiques gardent leur invariabilité à travers le temps chez les peuples les plus divers. La morale change au contraire selon les besoins de chaque époque. Si, comme le remarquait Pascal, ce qu'on appelle vice et vertu varie avec les climats, c'est que le vice et la vertu, étant l'expression des nécessités sociales d'une époque, se transforment forcément quand ces nécessités évoluent. Il est donc naturel qu'en matière sociale "la vérité en deçà des Pyrénées devienne erreur au delà ".

La caractéristique des natures primitives est de céder facilement à leurs impulsions. Il faut une longue éducation ancestrale pour apprendre au cerveau à dominer les impulsions des sens, et acquérir ainsi ce self-control que les Anglais considèrent comme une des plus importantes qualités du caractère.

L'homme vraiment moral n'a pas besoin de discuter sa morale avant d'agir. Une morale débattue demeure généralement sans force. 
Les canons restent des armes vaines quand ils ne sont pas soutenus par la force morale des combattants.

Un peuple ayant perdu son armature morale est bien près d'avoir tout perdu.

\section{IV - Les sources de la morale}

$\underline{\text { Retour à la table des matières }}$

La morale servant de guide dans la vie a de tout autres sources que celle enseignée par les livres.

La discipline externe, momentanément imposée par une force matérielle, disparaît avec cette force. Une discipline interne, fondée sur l'habitude, se maintient au contraire sans la nécessité d'une loi ou d'un maître.

Si la religion avait beaucoup d'influence sur la morale, les peuples les plus religieux seraient les plus moraux. Or, si l'on vante la religiosité des Espagnols et des Russes, personne ne loue beaucoup leur moralité.

Une morale ayant la crainte de l'enfer et l'espoir du paradis pour bases n'est qu'une forme un peu inférieure de la morale utilitaire. Les théologiens auraient dû le remarquer depuis longtemps.

La vertu ne reposant que sur la crainte de l'enfer et l'espoir du Paradis, est entièrement dépourvue de mérite. Les solitaires de Port-Royal obsédés par la terreur de la damnation suivaient des mobiles égoïstes ne méritant aucune considération.

Les disciplines purement rationnelles qu'on prétend généraliser aujourd'hui resteront toujours impuissantes à dominer les impulsions instinctives.

Quelles sont les bases possibles de la morale ? La peur des dieux ? Leurs châtiments sont lointains et peu redoutés aujourd'hui. La crainte des lois ? Elles s'éludent facilement. La raison? Il n'y a plus que de rares professeurs pour lui attribuer un tel rôle. La seule morale efficace est la morale inconsciente créée par les habitudes. Ces habitudes se développent à l'école, puis à la caserne, par une discipline d'abord 
sévèrement imposée, mais pratiquée ensuite sans effort grâce au mécanisme de la répétition.

Les universitaires qui, depuis Kant, prétendent édifier la morale sur une base à la fois rationnelle et mystique, au lieu de la fonder sur des habitudes issues de l'éducation, ne donnent qu'un enseignement totalement dépourvu d'efficacité.

La morale individuelle a pour soutien puissant la morale collective. Dans les manifestations de leur vie journalière, les hommes pensent et agissent généralement comme les autres membres du groupe professionnel, politique ou social auquel ils appartiennent. Leurs actes individuels sont alors régis par des influences collectives.

Le problème de l'organisation — industrielle ou sociale - représente surtout une question de discipline morale. Il échappe à l'action des institutions et des lois. 
Les incertitudes de l'heure présente

\section{Chapitre V}

\section{Les formes modernes du despotisme}

\section{I - L’extrémisme}

$\underline{\text { Retour à la table des matières }}$

L'extrémisme observé chez tous les partis révolutionnaires est un état mental où l'homme, dominé par une idée fixe, devient incapable de percevoir les réalités et leurs conséquences.

Les extrémistes de toutes opinions possèdent, malgré la divergence des buts poursuivis, des caractères identiques. L'extrémiste sincère est mystique, violent et borné.

Un extrémiste qui posséderait quelque trace de jugement et de clairvoyance cesserait aussitôt d'être extrémiste.

Le Conventionnel, pourvoyeur fervent de la guillotine, qui se déclarait prêt à transformer son pays en cimetière pour imposer ses croyances, traduisait la mentalité 
des extrémistes de tous les âges. Les apôtres du syndicalisme, du communisme et du socialisme rêvent les mêmes massacres.

La suggestion et la contagion mentale conduisent facilement une foule à l'extrémisme, mais cet extrémisme est généralement éphémère.

Dans les assemblées révolutionnaires, les opinions extrêmes tendent à engendrer des opinions plus extrêmes encore. Après les Girondins, ce fut la Terreur. Aux révolutionnaires russes modérés qui renversèrent le tsarisme, se substituèrent bientôt les sanguinaires bolchevistes.

Depuis les origines de l'histoire, tous les partis politiques extrêmes débutent dans la splendeur des illusions et finissent dans la bassesse des rivalités intestines.

Les progrès de certaines opinions extrémistes, visiblement absurdes, confirment cette fondamentale notion, que la force d'une théorie sociale ou religieuse ne dépend pas de sa valeur rationnelle, mais seulement de son empire sur les âmes.

L'ambition et le besoin de popularité ont pu conduire certains hommes clairvoyants à l'extrémisme; mais, sachant très bien que son application rendrait tout gouvernement impossible, ils le rejettent en arrivant au pouvoir.

À la phase ultime de son évolution, l'extrémisme ressort beaucoup plus du domaine de la pathologie mentale que de la politique. Les établissements d'aliénés sont remplis d'extrémistes.

\section{II - Le socialisme}

$\underline{\text { Retour à la table des matières }}$

Le socialisme, terme incertain, recouvre, suivant les races, des concepts très différents. Il en résulte que, dans leurs congrès, les socialistes de divers pays ne s'entendent guère.

Le socialisme aux États-Unis diffère totalement du socialisme européen. L'idéal du travailleur américain est de devenir patron, alors que l'ouvrier latin rêve surtout la suppression du patron. 
L'hypertrophie de certains sentiments est fort dangereuse pour un peuple. Celle de l'envie, fondement principal du socialisme, a déjà occasionné autant de ravages en Europe que les plus redoutables fléaux.

Si la jalousie, l'envie et la haine pouvaient être éliminées de l'univers, le socialisme disparaîtrait le même jour.

La haine des inégalités, base du socialisme, entraîne comme conséquence nécessaire la destruction des élites qui font la grandeur d'un pays. Le bolchevisme russe comprit parfaitement cette nécessité quand il procéda au massacre systématique des intellectuels. Le socialisme révolutionnaire qui triompha un instant en Allemagne et en Hongrie, répéta les mêmes destructions.

Le socialisme niveleur, très accessible à l'âme simpliste des foules, oriente facilement les forces aveugles et dévastatrices du nombre.

La haine intense des socialistes pour le capitalisme ne traduit souvent qu'une forme aiguë du désir de richesse. Les pays comme les États-Unis, où l'accès à la fortune est relativement facile, comptent peu de socialistes.

Les lois dites sociales ont un pouvoir destructeur parfois supérieur à celui des canons. C'est ainsi que l'application de la loi de huit heures à notre marine commerciale l'aurait rapidement ruinée au profit de concurrents trop intelligents pour accepter un tel décret. Il fallut l'abroger.

Supprimer, comme le voudraient les socialistes, la concurrence entre peuples et entre individus serait anéantir un grand facteur psychologique du progrès. Sans l'émulation, la plupart des découvertes dont bénéficie l'humanité n'eussent jamais vu le jour.

La population ouvrière des États-Unis montre expérimentalement que les classes déclarées ennemies par les socialistes ont beaucoup plus d'intérêt à s'associer qu'à se combattre. Si cette vérité pouvait s'établir en France, notre vie économique et sociale serait transformée.

L'initiative, le jugement, l'émulation, le goût du risque, qualités génératrices des grandes découvertes qui ont transformé le monde, sont des aptitudes exclusivement individuelles qu'aucune collectivité ne posséda jamais. Un pays où les socialistes réussiraient à ruiner l'effort individuel ne réaliserait plus un seul progrès.

Puisque des expériences répétées pendant de longs siècles d'histoire n'ont pas suffi à prouver que les progrès des peuples s'effectuent seulement par leurs élites, il était utile que la ruine de la Russie vînt le démontrer une fois encore. 


\section{III - Le syndicalisme}

$\underline{\text { Retour à la table des matières }}$

La Révolution avait divinisé la volonté du peuple. Nos révolutionnaires modernes l'exaltent aussi, mais déjà ils en séparent une sorte d'aristocratie qualifiée " prolétariat organisé et conscient ". En vertu de ce nouveau concept ils dédaignent la liberté, le suffrage universel et toutes les conquêtes démocratiques.

La discipline rigide acceptée par les adeptes du syndicalisme montre à quel point il deviendra despotique. On peut se demander si l'esclavage total de l'individu ne constitue pas l'aboutissement nécessaire de l'évolution démocratique.

Si les syndicats groupaient seulement des intérêts matériels similaires leur influence serait faible; mais, en associant des mécontentements et des haines, ils acquièrent une grande puissance révolutionnaire.

Les plus dangereux conflits de l'avenir ne se produiront pas toujours entre peuples rivaux. Ils éclateront entre syndicats d'un même peuple, rendus concurrents par leurs divergences d'intérêts. Les républiques syndicalistes italiennes du moyen âge, celle de Florence notamment, périrent tour à tour en de pareils conflits.

Si la dictature du prolétariat que réclament les socialistes devait être exercée par la totalité des travailleurs, elle ne différerait pas d'un gouvernement démocratique ordinaire. Pratiquée seulement par quelques individus, elle serait identique à celle des anciens régimes autocratiques.

Les théoriciens de la raison pure n'ont pas été heureux dans leurs tentatives pour l'appliquer au gouvernement des peuples. La première, celle de la grande Révolution, réalisée suivant l'évangile de Rousseau, aboutit aux massacres de la Terreur et à vingt ans de guerres. La seconde, celle de la Russie, inspirée par l'évangile de Karl Marx, amena la ruine totale du grand empire des tzars. 


\section{IV - Le communisme}

$\underline{\text { Retour à la table des matières }}$

Le bolchevisme communiste, comme le socialisme, a pour caractéristiques essentielles le mécontentement, la haine des supériorités et le désir de détruire violemment l'ordre de choses établi.

Quand, par une cause quelconque, le mécontentement grandit chez un peuple, il accepte d'emblée la première doctrine proposée pour remédier à ses maux. Le succès du communisme chez diverses nations est une conséquence de cette loi.

On trouve facilement des hommes disposés à massacrer, mais fort peu qui soient capables de diriger les mécanismes compliqués d'une civilisation. Les communistes russes ne soupçonnaient pas cette vérité élémentaire quand ils assassinèrent systématiquement les intellectuels de leur pays. Ils n'aperçurent leur erreur que devant la ruine économique qui résulta de ces hécatombes.

La désorganisation de la Russie, dont les ouvriers travaillent douze heures par jour sous la férule de maîtres très durs, pour un maigre morceau de pain, montre une fois encore combien les sociétés sont des organismes compliqués. Fils de la nécessité et du temps, ils sont aussi impossibles à transformer avec des décrets que la structure d'un être vivant.

Contrairement à ses théories, le communisme évolue de plus en plus vers un nationalisme jadis inconnu. Les Russes ne comprirent la force de l'idée de patrie que le jour où cette patrie parut menacée par l'étranger.

On a dit, avec raison, au Parlement anglais, que la Russie ne manquait pas d'argent, mais des cerveaux de l'Occident. L'expérience seule put faire entrer cette vérité dans la faible cervelle des communistes.

Si les théories communistes avaient régi l'humanité à travers les âges, l'homme vivrait encore au fond des cavernes, vêtu de peaux de bêtes et disputant aux animaux féroces une problématique pâture. La persistance du bolchevisme en Russie ramènerait bientôt ce pays aux ténèbres de la préhistoire. 
Une société purement bolcheviste n'a pas plus besoin de savants que les nègres du continent africain.

Le communisme russe a subi deux phases bien différentes. La première, l'égalisation générale, obtenue par le pillage des fortunes et le massacre des intellectuels. Ce fut l'âge d'or de la doctrine. Mais après l'épuisement des stocks pillés, lorsque, faute de capacités, les usines, les mines et les moyens de transports cessèrent d'être utilisables, l'illusion s'évanouit. il fallut alors solliciter les capitaux et l'intelligence de l'Occident.

L'extension du socialisme égalitaire, qui ravage la Russie, ramènerait l'Europe à la phase de barbarie qui suivit les anciennes invasions germaniques.

\section{V - L'égalité et le besoin de servitude}

$\underline{\text { Retour à la table des matières }}$

La liberté n'est, le plus souvent, pour l'homme que la faculté de choisir sa servitude.

Le premier ministre de l'Empire britannique assurait que " le monde entier était las de toutes les souverainetés ". En réalité, les peuples n'ont jamais autant demandé qu'aujourd'hui à être gouvernés. Les révolutionnaires eux-mêmes ne cessent de réclamer des dictateurs.

Le problème socialiste se ramène à savoir si l'égalité dans la misère, sans moyen d'en sortir, est préférable à des inégalités qui, permettant toutes les ambitions, constituent pour l'homme un stimulant énergique d'efforts et de progrès.

Quelles que puissent être les combinaisons rêvées, une dictature du prolétariat se ramènera toujours à la tyrannie absolue de quelques meneurs. Le résultat final d'un tel régime serait, comme en Russie, l'aggravation notable de l'ancien despotisme.

Si les bossus arrivaient à former la majorité d'une société, ils feraient probablement exterminer tous les individus se permettant de n'être pas contrefaits. C'est pour une raison du même ordre que les communistes russes supprimèrent tant d'intellectuels. 
Les incertitudes de l'heure présente

\section{Chapitre VI}

\section{L'évolution des civilisations}

\section{I - Comment les civilisations naissent et comment elles disparaissent}

$\underline{\text { Retour à la table des matières }}$

Les civilisations se fondent sur un petit nombre d'idées tenues pour des certitudes et universellement respectées. Ce n'est pas leur valeur rationnelle mais leur rôle qu'il importe de connaître.

Les grandes civilisations furent dominées chacune par un élément différent. Élément militaire dans la civilisation romaine; élément artistique et littéraire dans la civilisation grecque; élément religieux dans la civilisation du moyen âge; élément industriel dans le monde moderne.

La prédominance actuelle de la technique confère à l'ingénieur et à l'ouvrier une autorité comparable à celle des hommes d'Église pendant le moyen âge. 
Les peuples dont l'âme est stabilisée par un long passé possèdent seuls, malgré les divergences de partis, des opinions unanimes sur les questions fondamentales concernant les intérêts collectifs de leur race.

L'histoire des peuples civilisés retombés dans la barbarie, comme le monde romain après les invasions germaniques et la Russie de nos jours, révèle l'importance de certains éléments de civilisation, tels que le respect des contrats, de la propriété et de la vie des citoyens. Leur possession semble très naturelle. Pour les acquérir il fallut cependant des siècles d'efforts.

La civilisation crée forcément plus d'entraves à la liberté que l'état sauvage, mais il faut supporter ces entraves pour s'élever de la barbarie à la civilisation.

L'action des hallucinés dans le monde a été prodigieuse. De leurs idées dérive l'armature des grandes civilisations. Il n'est pas très sûr que la face du monde aurait changé, comme l'affirmait Pascal, si le nez de Cléopâtre eût été plus court. Il est certain que de tout autres formes de civilisations se fussent manifestées si de grands hallucinés comme Bouddha et Mahomet n'avaient pas possédé le merveilleux pouvoir de faire accepter par des millions d'hommes les illusions issues de leurs rêves.

Tous les grands empires qui ne furent pas anéantis par des conquêtes périrent sous l'influence des guerres civiles, c'est-à-dire se détruisirent eux-mêmes. Tel fut le sort de la Grèce dans le monde antique, des républiques italiennes au moyen âge, tel sera celui de la Pologne et probablement de l'Irlande dans les temps modernes.

Un peuple dont la population croît plus vite que ses moyens de subsistance finit toujours par envahir ses voisins. En s'emparant de toutes les colonies où l'Allemagne pouvait déverser l'excédent de ses habitants, l'Angleterre encercla au centre de l'Europe une nation amenée pour vivre à empiéter sur ses voisins, dès qu'elle se croira la plus forte.

Il est dangereux pour un peuple de compter dans son sein trop de vanités individuelles et pas assez d'orgueils collectifs.

À certaines heures de la vie des peuples, l'intelligence d'un seul homme peut changer leur destinée. L'Angleterre était sur le point de rayer la Turquie de l'Europe lorsque le génie d'un général sauva cet empire de l'abîme où il allait sombrer.

Les nouvelles conceptions politiques fondées sur le droit des nations à disposer d'elles-mêmes ne peuvent s'appliquer ni aux peuples amorphes, ne sachant pas au juste ce qu'ils veulent, ni à ceux qui sont incapables de vouloir longtemps la même chose. 
Les peuples, comme les individus, ne progressent que par des efforts continus. Quand leur évolution progressive s'arrête, une évolution régressive, créatrice de dégénérescence, lui succède bientôt.

\section{II - Les institutions politiques}

$\underline{\text { Retour à la table des matières }}$

Bien des révolutions seront, sans doute, encore nécessaires pour prouver que les changements d'institutions politiques ont une influence très faible sur la vie des nations. C'est la mentalité des peuples et non les institutions qui détermine leur histoire.

L'état actuel d'un être quelconque étant déterminé par la succession de ses états antérieurs, les transformations réalisables par chaque génération sont toujours minimes. L'état social d'un peuple se trouvant également conditionné par ses états antérieurs, les changements absolus que rêvent les partis politiques ne sont jamais réalisables.

La trame de la vie mentale d'un peuple est composée d'idées, d'habitudes, de croyances, de préjugés même, souvent dépourvus de valeur rationnelle. mais indispensables cependant à l'existence de ce peuple.

Pour changer les institutions d'un peuple, il faudrait d'abord transformer les sentiments et les rêves qui forment l'armature de son âme. L'impossibilité de telles modifications explique pourquoi les théoriciens du radicalisme n'ont jamais réussi à imposer nos institutions aux indigènes des colonies.

La dictature du prolétariat réclamée par les dirigeants des partis socialistes et syndicalistes signifie uniquement la possession, pour ces dirigeants, de tous les profits que l'exercice du pouvoir entraîne.

Dans les sciences, l'autorité des faits a depuis longtemps remplacé l'autorité des personnes. En politique, l'autorité personnelle reste toujours nécessaire.

L'unité d'un peuple peut être artificiellement créée par la force d'un chef, mais elle dépend alors de l'action de ce chef et ne lui survit pas. La seule unité durable est 
celle que réalise dans les âmes la communauté d'institutions, d'intérêts et de croyances.

Un parti politique prétendant dominer tous les autres finit par engendrer des réactions qui déterminent sa fin. Les croissants privilèges de la noblesse et du clergé amenèrent la révolution française. Les prétentions de l'Allemagne à l'hégémonie déclenchèrent la guerre où elle devait sombrer.

Au point de vue des institutions politiques possibles, les peuples peuvent être divisés en stables, instables et amorphes.

La Russie étant une masse amorphe de peuples primitifs sans intérêts communs, sans traditions et sans culture que recouvre une mince pellicule de civilisation, l'autocratie peut seule y établir l'unité.

Chez les peuples inférieurs, un pouvoir politique ne subsiste qu'en devenant absolutiste ou théocratique.

Les populations asiatiques ne supportant que des institutions autocratiques, le communisme russe fut bientôt obligé d'adopter un régime pratiquement identique à celui des anciens tsars.

En politique, l'action engendrant comme en mécanique une réaction égale et contraire, toute tyrannie grandissante crée rapidement une réaction destructrice de cette tyrannie.

\section{III - Quelques conséquences des idées démocratiques}

$\underline{\text { Retour à la table des matières }}$

Une démocratie se définit théoriquement : le gouvernement par le peuple. En réalité, aucune démocratie ne se maintient sans la direction d'une élite. Quand un gouvernement démocratique comme celui de l'Angleterre dure longtemps, c'est qu'il a fini par devenir une aristocratie de la fortune et de l'intelligence.

Le triomphe d'une démocratie marque souvent la fin du pays où il se produit. Sous les influences démocratiques, la Grèce sombra dans la servitude, Rome dans la 
décadence, les Républiques italiennes du moyen âge et la Russie moderne dans l'anarchie et la dictature.

Platon soutenait que tous les progrès de l'esprit humain sont dus à l'aristocratie de l'intelligence. Contrairement à cette doctrine, les dictateurs russes divisèrent les hommes en quatre classes, dont la plus haute se trouvait représentée par les ouvriers manuels et la plus basse par les intellectuels. Une ruine totale montra vite les conséquences de cette classification.

L'expérience russe aura définitivement prouvé qu'un gouvernement bourgeois, si médiocre qu'on le suppose, est infiniment moins despotique qu'un gouvernement prolétarien, si parfait qu'il puisse être.

Loin de réduire les haines internationales, le progrès des démocraties les rend chaque jour plus fortes.

Une des plus fréquentes erreurs du régime parlementaire consiste à renverser les dirigeants au moment précis où les événements, dominant les volontés, ne permettent pas aux successeurs des ministres renversés de rien changer à la politique de leurs prédécesseurs.

Le principe du service universel obligatoire substituant aux petites armées de jadis la totalité des habitants valides d'un pays, est évidemment parfait au point de vue démocratique. Dans la pratique, il engendre de véritables guerres d'extermination et, par conséquent, la destruction des démocraties créatrices de ce principe.

L'âge moderne se trouve obligé de faire vivre ensemble des hommes exigeant l'égalité, alors que les progrès des civilisations, comme ceux de la nature, se réalisent seulement par des inégalisations successives.

\section{IV - Les récits historiques}

$\underline{\text { Retour à la table des matières }}$

Les historiens se mettraient peut-être d'accord si les événements ne présentaient qu'une seule face; mais comme ils en ont plusieurs, susceptibles d'interprétations diverses, l'accord devient impossible.

Les livres d'histoire révèlent surtout les croyances de leurs auteurs. 
Si l'histoire est une science très conjecturale, ce n'est pas seulement en raison de notre faible connaissance des événements, mais aussi parce que les sentiments qui les déterminèrent restent ignorés.

Certains événements comme la Saint-Barthélemy semblent incompréhensibles, parce que nous n'éprouvons plus les sentiments qui les firent naître. Il faudrait posséder la mentalité de l'époque pour comprendre l'enthousiasme provoqué par ce massacre dans l'Europe catholique. De nombreuses médailles furent frappées, notamment par Grégoire XVI, pour fêter l'événement. Les tableaux que fit exécuter ce pape pour en perpétuer les détails figurent toujours au Vatican.

Les narrations historiques sont tellement incertaines qu' on y rencontre les mêmes erreurs répétées indéfiniment. Quelques auteurs ayant assuré que l'empire byzantin constituait une période de décadence, tous les écrivains l'ont redit. Il fallut les ressources de l'érudition moderne pour démontrer que l'Empire byzantin posséda pendant mille ans une des plus brillantes civilisations de l'histoire.

On a justement fait observer que les guerres modernes ruinent autant le vainqueur que le vaincu.

Il ne faudrait pas, cependant, les croire inutiles, puisque quelques batailles suffisent parfois pour transformer entièrement les conditions d'existence d'un peuple. C'est par sa lutte avec la Russie que le Japon devint une grande puissance. C'est par sa guerre avec la Grèce que la Turquie, sur le point de disparaître, reprit son ancienne puissance. C'est encore par la guerre que fut transférée à l'Angleterre l'hégémonie germanique.

En politique, les principes théoriques déduits de la raison pure créent facilement des désastres. Le principe d'équilibre résultant de la lente action des siècles avait amené l'Europe à un certain état de stabilité. Le nouveau principe théorique des nationalités la conduira fatalement à des guerres répétées jusqu'à ce que des équilibres nouveaux soient établis.

Des ententes provisoires sont supérieures aux alliances parce qu'une alliance, quelle que soit sa forme, ne survit pas à l'évanouissement des intérêts qui la firent naître.

Le grand talent des historiens doués de prestige est de rendre vraisemblables les invraisemblances de l'histoire.

Les découvertes de la psychologie suffisent à montrer que l'histoire classique est le récit d'événements aussi incompris de leurs auteurs que des écrivains qui les racontèrent. 
L'étude de l'histoire ne semble pas donner aux historiens une grande faculté de prévision. On sait avec quelle ardeur beaucoup de professeurs, et surtout Renan, formaient jadis des vœux pour la réalisation de l'unité allemande qui nous valut les guerres de 1870 et de 1914 . 
Les incertitudes de l'heure présente

\section{Chapitre VII}

\section{L'intelligence, le caractère et l'éducation}

\section{I - L’incompréhension et les conflits de mentalités}

$\underline{\text { Retour à la table des matières }}$

Les mêmes mots pouvant évoquer des idées différentes, la communauté de langage n'implique nullement celle des pensées. L'incompréhension domine les relations entre individus de sexe, d'éducation, de race, différents.

Comment espérer une communauté de pensée quand on voit les plus usités des termes abstraits Dieu, âme, nature, liberté, etc., évoquer des conceptions très diverses suivant la mentalité des êtres qui les entendent?

Vouloir interpréter au point de vue rationnel un sentiment ou une croyance, c'est s'interdire de les comprendre. Le rationnel dont le rôle se montre si grand dans la genèse des découvertes exerce une très faible influence dans la vie des peuples. 
La compréhension d'un code, d'une institution, d'un traité, variant avec les passions, les croyances, les préjugés de chaque époque, les interprétations des historiens changent constamment.

La jeunesse se montre toujours intolérante parce que, n'ayant ni le sens des possibilités ni celui des nécessités, elle croit facile de réformer ce qui choque sa logique rationnelle. Il faut réfléchir longtemps pour découvrir que ce n'est pas cette logique qui mène le monde.

Les contes, les légendes, les oeuvres d'art, les romans même, sont beaucoup plus véridiques que les livres d'histoire. Ils expriment la sensibilité d'une époque, alors que le langage rationnel des historiens ne la fait pas connaître.

Notre opinion des choses doit naturellement varier avec l'évolution de ces choses. L'ignorant seul possède des opinions invariables.

Si l'incompréhension domine les relations entre peuples, c'est que la plupart des questions impliquent des points de vue divers : rationnel, sentimental et politique n'ayant pas de commune mesure.

La sympathie naît facilement entre nations éloignées ne se connaissant pas. Dès qu'elles se trouvent en contact, leurs divergences de sentiments, d'idées et de croyances éclatent et toute sympathie s'évanouit.

Avec l'interdépendance économique croissante des peuples, l'Europe cessera bientôt d'être le centre du monde. Elle ne l'est plus militairement depuis que l'expérience a prouvé qu'une armée pouvait facilement franchir l'Océan. Elle ne l'est plus scientifiquement depuis que les inventions du nouveau monde égalent celles de l'ancien. Elle ne l'est plus économiquement depuis que la majeure partie de l'or européen a passé en Amérique.

Il est aussi difficile de vivre avec les hommes ne changeant jamais d'idées qu'avec ceux qui en changent constamment.

Des événements comme ceux de la grande guerre restent toujours mal compris quand on les isole de leurs causes passées et de leurs effets futurs. 


\section{II - Le caractère et l'intelligence dans la vie des peuples}

$\underline{\text { Retour à la table des matières }}$

L'homme s'avoue rarement les sentiments qui le mènent et que, d'ailleurs, il ne connaît pas toujours. Les révolutionnaires extrémistes, dont la haine et l'envie sont les vrais guides, se croient animés du désir d'établir le règne de la justice et du bonheur.

Les erreurs intellectuelles sont généralement sans durée. Les erreurs d'origine sentimentale ou mystique persistent, au contraire, longtemps et réussissent parfois à bouleverser le monde.

Parmi les facteurs actuels de la vie des peuples l'envie est le plus fort, l'amour du prochain le plus faible, l'espérance le plus incertain.

L'amitié représente un sentiment faible, mais durable; l'amour, un sentiment fort, mais peu durable. L'envie, dont le rôle social devient si prépondérant, constitue un des rares sentiments possédant à la fois force et durée.

On trouve plus facilement mille hommes prêts à obéir qu'un seul capable de prendre une initiative.

Le plaisir de tuer, qui anime les chasseurs, est si grand qu'on l'offre avant tout autre aux souverains visitant un pays étranger.

Ne nous plaignons pas trop de voir l'hypocrisie gouverner les hommes. Le monde deviendrait vite un enfer si l'hypocrisie en était bannie.

On croyait jadis que la science adoucirait les mœurs. L'expérience prouve, au contraire, qu'elle a rendu les guerres beaucoup plus féroces et meurtrières que celles du passé.

Pour doser les sentiments, nous ne possédons encore aucun moyen de mesure. Le passage du qualitatif au quantitatif, qui transforma toutes les sciences, reste à réaliser dans le cycle de l'affectif. 
Si la science arrivait à découvrir un thermomètre des sentiments, des passions et des volontés, la conduite de l'homme dans une circonstance donnée serait aussi facile à calculer que la trajectoire d'une planète.

La civilisation réelle est celle des sentiments, disait un Japonais. On pourrait ajouter que si quelques années d'enseignement universitaire procurent une dose suffisante de civilisation intellectuelle, il faut beaucoup plus de temps pour civiliser les sentiments. Les cruautés et les dévastations des Allemands pendant la guerre ont de nouveau montré à quel point la civilisation de l'intelligence reste étrangère à celle des sentiments.

Le fondement principal de la grandeur d'un peuple ne réside ni dans le chiffre de ses habitants, ni dans l'étendue de son territoire, ni dans le nombre de ses canons, mais dans la force de son caractère.

Une volonté forte est beaucoup plus utile dans la vie qu'une instruction forte superposée à une volonté faible.

Loin d'être une preuve de caractère, la violence constitue souvent une manifestation de faiblesse. L'homme faible se montre parfois violent pour cacher sa faiblesse.

L'être qui ne sait pas dominer ses impulsions instinctives devient facilement esclave de ceux qui lui proposent de les satisfaire.

Dans toutes les affaires humaines il faut risquer pour réussir. C'est de la juste évaluation des chances de gain et de perte que dépendent les grands succès.

Bien des événements de la dernière guerre ont prouvé quels désastres peuvent causer l'indécision et l'absence d'initiative. Il a été reconnu devant le Parlement anglais que la guerre eût été très brève si, au début des hostilités, les navires alliés avaient eu à leur tête un amiral assez hardi pour entrer à Constantinople comme le firent les Allemands. Pour tâcher de réparer cette insuffisance du caractère, 100.000 hommes périrent inutilement dans l'expédition des Dardanelles.

Dans les civilisations compliquées où la moindre profession se hérisse de concours mnémoniques éliminatoires, il arrive fatalement que des capacités réelles ne peuvent se libérer de ces artificielles barrières. Ainsi se produisent des pertes de forces et des haines de classes inconnues dans les pays neufs comme l'Amérique, où ces entraves sont ignorées.

Un sentiment fort ne pouvant être dominé que par un sentiment plus fort, on comprend l'usage de la terreur par les conquérants et les fondateurs de croyances. Les codes civils ou religieux eurent toujours de terrifiantes menaces pour soutiens. 
Pour agir sur les êtres qui nous entourent, la connaissance de leurs défauts est parfois plus utile que celle de leurs qualités.

Une des grandes causes de faiblesse des peuples latins tient à ce que tout leur personnel dirigeant est issu d'examens universitaires prouvant la mémoire des candidats, mais nullement les qualités de caractère qui font la valeur de l'homme dans la vie.

\section{III - L'intelligence, les sentiments et l'intuition}

$\underline{\text { Retour à la table des matières }}$

L'intelligence et les sentiments sont des compagnons inséparables, mais qui, depuis l'origine des âges, ont rarement réussi à s'entendre.

L'intelligence ayant considérablement évolué dans le cours des siècles, alors que les sentiments ont peu changé, il en résulte une discordance croissante entre la logique sentimentale, qui détermine la conduite, et la logique rationnelle, qui cherche à la diriger, mais y réussit rarement.

Dans le domaine de l'intelligence, la différence entre les hommes est immense. Dans les cycles de l'affectif et du mystique où s'élaborent les causes de nos actions, l'inégalité disparaît. C'est pour cette raison qu'un bolcheviste sans culture et un professeur instruit peuvent accepter des illusions identiques.

La raison se met facilement au service des sentiments, alors que ces derniers se mettent rarement au service de la raison. Cette loi psychologique explique l'origine de guerres qu' aucun argument rationnel ne pourrait justifier.

L'être dont l'intelligence ne réussit pas à dominer la sensibilité pourra devenir un remarquable artiste, un éminent écrivain, mais non un homme d'État supérieur.

Une conviction fondée seulement sur la raison devient rarement mobile d'action. Les influences mystiques et sentimentales sont indispensables pour faire agir. 
L'homme est en progrès quand il commence a séparer sa raison de ses passions. Le progrès s'accentue lorsque sa raison devient assez forte pour dominer un sentiment présent en lui opposant l'influence d'un sentiment lointain.

L'acquisition de l'aptitude à dominer ses sentiments exigea des siècles d'efforts. Bien des peuples en sont restés à l'âge où Esaü vendait contre un plat de lentilles présent son droit d'aînesse futur.

Grâce au mode de connaissance qualifié intuition, les femmes arrivent à deviner des conséquences que la logique rationnelle ne perçoit pas aisément.

La raison seule peut montrer si les certitudes intuitives constituent des réalités ou des erreurs. L'intelligence est donc le complément nécessaire de l'intuition.

Les intuitions intellectuelles sont génératrices de découvertes dans tous les domaines de l'art et de la pensée. Les intuitions sentimentales représentent les véritables guides dans les circonstances difficiles de la vie.

On reconnaît qu'une poussière d'hommes est devenue une nation lorsque, dans les grands événements comme la dernière guerre, se forment instantanément des intuitions collectives conduisant tous les citoyens à une conduite identique malgré les divergences de croyances.

L'habitude, permettant de canaliser les intuitions et refréner les impulsions, constitue un guide de la vie plus sûr que tous les enseignements des livres.

\section{IV - L'instruction et l'éducation}

Retour à la table des matières

Dans les discussions sur la réforme de l'enseignement, un éminent orateur assurait que "l'hellénisme donne à l'esprit l'équilibre et l'harmonie d'un temple ". Cet équilibre de l'esprit ne suffit pas, sans doute, à créer l'équilibre des sentiments puisque les permanentes dissensions des anciens Grecs les conduisirent à la servitude.

Chaque phase d'une civilisation, différant des phases antérieures, nécessite une éducation adaptée aux besoins nouveaux et aux mentalités nouvelles. C'est méconnaître cette vérité banale qu'imposer à la jeunesse actuelle la culture gréco-latine du monde antique et du moyen âge. 
Les défauts d'un peuple n'étant visibles qu'aux étrangers ne se corrigent guère. Tous connaissent l'infériorité de nos méthodes universitaires, mais comme nous ne l'apercevons pas, aucune réforme réelle n'est possible.

La nourriture intellectuelle donnée par l'instruction est comparable à la nourriture matérielle. Ce n'est pas ce qu'on mange qui nourrit, mais seulement ce qu'on digère.

Beaucoup de nos idées sociales seront transformées lorsqu'on découvrira qu'un ouvrier habile est intellectuellement fort supérieur à un bachelier médiocre.

Il n'est d'éducation utile que celle cultivant les aptitudes spéciales de chaque être. On obtient alors tout ce que l'élève peut donner sans exiger un inutile travail.

En imposant à tous les élèves une instruction identique, on obtient un minimum de rendement avec un maximum d'effort.

Une des erreurs latines qui ont le plus pesé sur la vie de nos colonies fut de croire que l'instruction classique permettait de franchir rapidement les étapes séparant la barbarie de la civilisation. Un nègre peut recevoir une éducation classique complète sans devenir un homme civilisé.

Les expériences permettant de remplacer la tête d'un insecte par celle d'un autre insecte de sexe différent ont montré que les femelles à tête de mâle prennent les instincts des mâles. On peut se demander si l'éducation masculine donnée au sexe féminin ne réalisera pas indirectement cette substitution.

Les méthodes propres à la culture de l'intelligence sont diverses. Pour agir sur le caractère, il n'en existe qu'une : la pratique des qualités à développer. L'initiative, la persévérance et la volonté ne s'acquièrent pas autrement.

Notre enseignement universitaire se transformera seulement quand il sera généralement admis que les livres ne suffisent pas à éduquer le caractère, la morale et l'intelligence.

C'est avec raison que les Anglais et les Américains attribuent une forte influence éducatrice aux jeux sportifs. Ces jeux enseignent, notamment, l'art d'obéir, sans lequel ne saurait s'acquérir l'art de commander. 
Les peuples ayant découvert que l'éducation du caractère est plus importante que celle de l'intelligence sont très en avance sur ceux qui n'ont pas fait cette découverte. Les universités latines ne l'ont pas réalisée encore.

La grande habileté de la Prusse fut de transformer, grâce à son système d'éducation pratique et à sa discipline militaire, une poussière d'individualités médiocres, provenant de races diverses, en un bloc collectif très fort.

Après avoir constaté que, depuis la paix, le goût du travail, l'honnêteté professionnelle et la politesse disparaissaient de l'Allemagne, une revue germanique attribuait très justement cette décadence à ce que la jeunesse allemande n'est plus soumise à la discipline militaire.

Un système quelconque d'instruction ou d'éducation est parfait s'il réussit à créer des automatismes inconscients utiles. L'intelligence possède alors des serviteurs dociles prêts à exécuter ses ordres. Mal dressés, ils ne les exécutent pas.

La discipline peut remplacer bien des qualités. Aucune ne remplace la discipline.

L'intelligence est un vernis qui recouvre les sentiments mais ne les transforme pas.

Le temps et l'habitude usent rapidement l'amour mais fortifient au contraire l'amitié, même quand elle succède parfois à l'amour.

Le jugement sans volonté est aussi inutile que la volonté sans jugement. 
Les incertitudes de l'heure présente

\title{
Chapitre VIII
}

\section{Les influences conscientes et} inconscientes dans la vie des peuples

\author{
I - La vie consciente \\ et la vie inconsciente
}

$\underline{\text { Retour à la table des matières }}$

La vie inconsciente est à la vie consciente ce qu'est une montagne à un grain de sable, la mer profonde aux vagues qui la recouvrent. Son étude éclaire déjà d'une lumière toute nouvelle les mobiles de la conduite.

C'est la volonté inconsciente qu'il faut influencer pour déterminer les hommes à l'action. L'âme consciente peut être convaincue par persuasion, mais la conviction seule ne suffit pas à faire agir.

Les causes de certains événements historiques, les origines de la grande guerre notamment, demeurent incompréhensibles quand on ignore les différences qui séparent la volonté consciente de la volonté inconsciente. 
La substitution de la pensée collective à la pensée individuelle représente une des caractéristiques de l'âge actuel. Les hommes capables de pensées personnelles deviennent chaque jour plus rares. En politique ils ont à peu près disparu.

C'est parce que les influences collectives agissent profondément sur l'inconscient, que la part d'indépendance possible à l'individu isolé devient fort restreinte.

Ce n'est pas toujours la mauvaise foi qui empêche de conformer les actes aux discours. Les actes importants traduisent les impulsions inconscientes de l'âme ancestrale, alors que les discours dérivent de l'âme consciente individuelle. Si, en 1914, les socialistes qui avaient juré de déserter en cas de guerre rejoignirent le front sans hésiter, c'est que l'âme inconsciente de la race qui dirigea leur conduite fut plus forte que la raison consciente inspirant leurs discours.

En politique, des mobiles inconscients dirigent souvent les actes que leurs auteurs croient dictés seulement par la raison. Le projet de démembrer la Turquie, qui souleva tout l'Islam contre l'Angleterre, eut pour principal auteur un pieux ministre anglais inconsciemment guidé par l'idée d'une revanche de la croix sur le croissant.

Les grands bouleversements font naître des idées inconscientes qui, substituées à celles servant habituellement de guide, engendrent des mouvements sociaux imprévus.

Une opération intellectuelle consciente suffisamment répétée, passe dans l'inconscient et devient habitude. Maintenue pendant plusieurs générations, elle finit par constituer un caractère de race.

Une décision réfléchie ne conduit pas toujours à l'action. L'immense majorité des hommes agit au contraire sous l'influence d'impulsions inconscientes dont toute réflexion est exclue.

Il n'est de discipline réelle que la discipline devenue inconsciente. Celle qui repose uniquement sur la contrainte est sans durée et sans force.

Les habitudes inconscientes ont une force que ne possèdent jamais les principes. 


\section{II - La vie collective et le rôle des meneurs}

$\underline{\text { Retour à la table des matières }}$

Les foules et les individus de mentalité inférieure possèdent ce caractère commun d'être fortement influencés par les événements présents et très peu par leurs conséquences, si inévitables qu'elles puissent être.

L'erreur individuelle est tenue pour vérité dès qu'elle devient collective. Aucun argument rationnel ne peut alors l'ébranler.

Perdue dans les rouages complexes des civilisations modernes, enveloppée d'effets dont les causes lui échappent, la foule attribue forcément à des volontés particulières les événements dont elle ne peut saisir les lois. Ses révoltes n'ont souvent pas d'autres causes.

Dans les grands événements intéressant la vie d'un peuple, l'homme agit surtout sous des influences collectives. Son égoïsme individuel s'évanouit alors au point de lui faire sacrifier sans hésiter sa vie à des intérêts communs.

Les hommes se divisent en meneurs et en menés. L'immense majorité se compose de menés.

Une collectivité n'a d'autre cerveau que celui de son meneur.

Croyances politiques et croyances religieuses ont un même mécanisme de propagation. L'affirmation, la répétition, le prestige et la contagion suffisent à créer des suggestions auxquelles les collectivités résistent rarement.

Les plus évidentes vérités restent sans action sur l'âme des multitudes quand elles manquent d'apôtres pour les propager.

Les illusions que d'ambitieux meneurs font surgir dans l'âme populaire deviennent souvent redoutables. Si l'illusion des capacités politiques et industrielles du prolétariat, qui a déjà ruiné la Russie, continuait à se répandre, elle amènerait la fin des plus brillantes civilisations. 
Un meneur doué de prestige n'a pas besoin de donner des explications. Les ordres expédiés au Congrès socialiste de Tours par les dictateurs de Moscou et dont ils obtinrent la respectueuse acceptation étaient formulés en termes impérieux et brefs ne contenant aucun exposé de doctrine.

La contagion mentale peut se produire sans l'intervention personnelle d'un meneur. Un mot, une formule, un courant d'opinion suffisent parfois à suggestionner une multitude.

La mentalité grégaire des foules permettra toujours aux meneurs d'imposer une doctrine quelconque. Les plus absurdes croyances ne manquèrent jamais d'adeptes.

Le despotisme des meneurs de la classe ouvrière dépasse de beaucoup celui des tyrans asiatiques. Aucun de ces derniers n'aurait osé ordonner l'arrêt total des chemins de fer, imposé en Angleterre pendant un mois par les chefs syndicalistes, ni la suppression des journaux imposée en France pendant trois semaines par quelques meneurs.

\section{III - L’âme des peuples}

$\underline{\text { Retour à la table des matières }}$

La vie politique d'un peuple reste incompréhensible quand on ignore combien les sentiments individuels sont différents des sentiments collectifs.

L'âme d'un peuple stabilisée par un long passé finit par posséder des éléments aussi fixes que les caractères anatomiques. Aucune éducation ne peut alors lui faire subir des transformations profondes.

La connaissance du caractère d'un peuple, de sa morale, des idées qui le guident et de l'éducation qu'il reçoit, révèle facilement s'il est sur la voie du progrès ou sur celle de la décadence.

Parmi les facteurs déterminant la force des Anglo-Saxons, aussi bien en Angleterre qu'en Amérique, il faut citer: le self-control et le respect des lois. Des siècles d'efforts sont parfois nécessaires pour acquérir ces qualités. Elles ne s'apprennent pas dans les livres. 
Avec une dépêche anodine dont il altéra le sens, Bismarck provoqua en France une explosion d'indignation qui conduisit le gouvernement à déclarer la guerre sans vérifier l'exactitude de cette dépêche. Il fallait bien connaître la grande émotivité du peuple français pour réussir une telle opération. Son succès eut été probablement nul en Angleterre.

Ce fut surtout parce qu'ils avaient perdu leur discipline sociale que les Grecs, dont la pensée et les arts charmèrent le monde, furent asservis par des Romains ne possédant qu'une faible culture, mais dont la discipline avait unifié les âmes.

Dans les grands événements menaçant l'existence d'un peuple, la volonté des morts soutient énergiquement celle des vivants. Les nations n'ayant pas assez de morts pour les défendre ne résistent guère. Tel fut Je cas de la Russie vers la fin de la grande guerre.

La plupart des hommes, surtout quand ils font partie d'une collectivité, éprouvent le besoin d'être dirigés dans leurs moindres actes. Ce besoin de servitude est un des principaux éléments de succès du socialisme.

Dans l'immense majorité de leurs actes, les peuples sont dirigés par des habitudes et des croyances. Dans les circonstances où ces mobiles n'agissent plus, les illusions créées par les hasards du moment deviennent les seuls guides.

Les découvertes individuelles transforment les civilisations. Les croyances collectives régissent l'histoire.

La grande force des décisions collectives réside dans le pouvoir mystique que le nombre exerce sur l'âme des multitudes. C'est pour cette raison que les chefs d'État sont obligés de paraître s'appuyer sur l'opinion populaire.

Une des bases les plus efficaces de l'éducation morale est la contagion mentale résultant de l'influence du milieu. Le vice aussi bien que la vertu se propagent par contagion. 


\section{IV - Les oscillations de l'opinion}

$\underline{\text { Retour à la table des matières }}$

Les lois de la genèse des opinions permettent seules de comprendre comment devint belliqueuse la mentalité pacifique d'Américains désireux de rester neutres pour commercer fructueusement avec les belligérants. Sans doute l'hégémonie allemande menaçait leurs futurs intérêts. Mais comment ces intérêts lointains devinrent-ils visibles ? La guerre sous-marine ne fut qu'une des causes de la transformation des sentiments qui détermina l'envoi d'un million de soldats en Europe.

Tous les hommes sont plus ou moins suggestionnables, mais ils le sont surtout dans les sujets qu'ils ne connaissent pas. Ainsi s'explique la crédulité de nombreux savants.

Avec les moyens actuels de publicité, une opinion une croyance, une doctrine, peuvent être lancés comme un produit pharmaceutique quelconque. Ce fut par leur propagande que les communistes russes recrutèrent tant d'adeptes à l'étranger.

Si la publicité des journaux constitue un moyen de persuasion très efficace, c'est que peu d'esprits se trouvent assez forts pour résister au pouvoir de la répétition. Chez la plupart des hommes elle crée bientôt la certitude.

Les influences déterminant le vote d'un électeur sont bien rarement d'origine rationnelle. Des haines, des craintes, des espérances, provoquent généralement son choix.

En matière scientifique, pour être cru il faut prouver. En politique, les discours d'un orateur doué de prestige suffisent à créer d'imaginaires certitudes.

La presse canalise l'opinion beaucoup plus qu'elle ne la dirige. Elle sert aussi à condenser en termes nets des milliers de petites opinions fragmentaires trop incertaines pour être clairement formulées.

C'est parce que la répétition transforme facilement l'erreur en vérité que fut acceptée dans les milieux militaires la croyances au respect de la neutralité belge par les Allemands. Elle nous coûta l'invasion de dix départements.

Les événements capables d'agir violemment sur la sensibilité collective produisent ce qu'on appelle une explosion d'opinions, c'est-à-dire l'orientation instantanée 
d'émotions collectives dans le même sens. Ainsi naissent les révolutions. Telle, par exemple, celle du 4 septembre 1870 qui renversa instantanément l'Empire. Telle encore la révolution allemande qui, au moment de l'armistice, contraignit l'empereur d'Allemagne et tous les souverains germaniques à une abdication immédiate.

C'est s'illusionner sur les hommes d'État que s'imaginer qu'ils apporteront dans leurs actes l'énergie manifestée dans leurs discours.

Si les peuples sont souvent déçus par leurs gouvernants, c'est qu'ils leur demandent de réaliser le meilleur, alors qu'un homme d'État ne peut réaliser que le possible.

Pour beaucoup d'êtres la vie serait parfois bien lourde si la nature ne leur avait donné la faculté de parler sans réfléchir et d'émettre des opinions dépourvues de tout fondement.

C'est du besoin de récriminer que dérivent la plupart des opinions populaires. Pour beaucoup d'esprits, récriminer représente un grand bonheur, souvent même le seul bonheur. 
Les incertitudes de l'heure présente

\section{Chapitre IX}

\section{L'évolution des dieux} dans l'histoire

\section{I - Le rôle des dieux}

$\underline{\text { Retour à la table des matières }}$

L'histoire des peuples est dominée par celle de leurs dieux. Dans les temps modernes cette domination est restée aussi grande, mais les divinités ont changé de nom. Elles sont remplacées par des idées et des formules auxquelles leurs adorateurs attribuent la même puissance qu'aux anciens dieux.

Aucun peuple ne vécut sans dieux. L'usure du temps, et non la raison, quelquefois les renverse, mais leur trône ne reste jamais vide. Le paganisme usé fit place au Christianisme, qui, usé à son tour, tend à être remplacé par la foi socialiste.

Une civilisation entièrement dégagée d'influences mystiques serait-elle viable ? Nous l'ignorons. Aucune civilisation semblable ne s'est encore manifestée à la surface du globe. 
Bien que les croyances religieuses n'aient eu que des illusions pour soutien, elles ont servi d'armature aux grandes civilisations. Pour les propager ou les combattre, le monde fut souvent bouleversé.

Toutes les grandes divinités de l'histoire : Jupiter, Jéhovah. Bouddha, Allah et tant d'autres que des millions d'hommes ont adorées ou vénèrent encore ne furent pas des créations de la peur, comme le voulait Lucrèce. Elles naquirent de l'espérance, seule divinité que le temps n'ait pu ébranler.

Les dieux étant issus des mêmes illusions, et ayant exercé le même rôle, on ne saurait établir de hiérarchie entre eux.

Les croyants imaginent toujours à leur image le dieu qu'ils adorent. Les Hindous pacifiques attribuent à Bouddha leur tolérance et leur douceur. Les Carthaginois, les Chrétiens et les Juifs dotèrent leurs Dieux de l'esprit vindicatif qui les animait.

Un des plus utiles rôles des religions fut de créer des certitudes d'existence future capables d'embellir la vie présente. L'homme assuré d'un bonheur éternel est plus heureux que s'il croit son existence éphémère. Seule, la peur de l'enfer l'empêche d'être tout à fait heureux.

C'est principalement dans la manifestation des arts plastiques que les religions montrèrent leur influence. Les grandes oeuvres d'art de l'Égypte, de l'Inde et de l'Europe furent surtout des monuments religieux. A des dieux supposés éternels il fallait bien construire des temples également éternels.

Une des forces des dieux réside dans la difficulté de les remplacer. Les illusions socialistes sont encore moins satisfaisantes pour l'esprit que les illusions religieuses.

La psychologie explique la propagation des croyances religieuses, mais la naissance des dieux est d'une interprétation beaucoup plus difficile. Comment naquirent : Moloch, Jupiter, Apollon, Jéhovah et bien d'autres ? Certaines religions, comme l'islamisme, sortirent tout entières du cerveau d'un halluciné, mais cette genèse n'est pas applicable à l'histoire de tous les dieux.

Ce sera seulement sans doute avec les derniers hommes que disparaîtront les derniers dieux. 


\section{II - Le pouvoir des croyances}

$\underline{\text { Retour à la table des matières }}$

L'homme dominé par une croyance voit son énergie notablement accrue. La foi constitua toujours un très puissant mobile d'action. On a dit justement qu'elle peut soulever des montagnes.

La force d'une croyance telle que l'islamisme ou le bolchevisme ne dépend pas des dogmes qu'elle enseigne, mais de l'énergie des convictions qu'elle inspire.

L'unité de pensée, qui confère une grande force à un peuple, ne fut guère fondée jusqu'ici que sur des croyances religieuses. La raison seule n'a pas encore réussi à solidariser les hommes.

C'est surtout quand une religion est morte ou va mourir que son utilité apparaît. Plusieurs peuples ont vu leur civilisation périr ou se transformer avec la mort de leurs dieux.

Alors même que l'usure du temps fait disparaître une croyance, elle conserve longtemps encore dans les âmes une influence assez forte pour devenir mobile d'action. La haine de certains diplomates anglais puritains à l'égard des Musulmans n'a probablement pas d'autres causes.

La faim et l'amour ne suffiraient pas à soutenir la vie du monde, comme l'affirmait un grand poète. Il faut y ajouter l'espoir créé par les croyances.

Les dieux vindicatifs condamnant leurs créatures à des supplices éternels pour des fautes légères eurent une utilité certaine aux âges de grande barbarie. A une époque éclairée, leur férocité n'est plus défendable.

\section{III - Les formes diverses de croyances}

Retour à la table des matières

Les adeptes des grandes religions monothéistes ne sauraient être tolérants. Détenteurs de la vérité pure, ils considèrent comme un devoir de massacrer les infidèles pour extirper l'erreur. Si le protestantisme est devenu tolérant, c'est que sa division en sectes nombreuses l'a rapproché du polythéisme. 
Les religions polythéistes furent toujours tolérantes. Les croisades, les guerres de religion, l'inquisition auraient été impossibles chez les peuples polythéistes. Si Rome, dont la tolérance religieuse était complète, finit par persécuter les Chrétiens, c'est qu'ils cherchaient à ébranler son pouvoir politique.

Si le fait d'être monothéiste constituait une supériorité pour une religion, il faudrait mettre l'islamisme au premier rang. Il représente, en effet, la seule religion vraiment monothéiste. Le Jéhovah des Juifs avait des rivaux qu'il ne domina jamais complètement. Le Dieu des Chrétiens comprend trois personnes et une armée de saints, divinités secondaires comparables à celles du paganisme.

Les fondateurs de religions ont sagement agi en dotant leurs dieux de toutes les passions animant les hommes. Si ces dieux sont jaloux, irritables et vindicatifs, c'est que, représentés autrement, ils n'eussent séduit personne.

Tanit, à Carthage, ordonnait des immolations d'enfants. Les dieux d'Homère exigeaient qu'Agamemnon leur sacrifiât sa fille. Les idées modernes héritières des anciens dieux réclament encore plus d'hécatombes. A l'époque de la Révolution les divinités nouvelles désignées sous les noms de Liberté et d'Égalité ensanglantèrent vingt ans l'Europe.

On ne compte plus les massacres engendrés par l'idée communiste.

Dans le domaine de la foi religieuse l'absurde est inconnu. On ne citerait pas au cours de l'histoire une seule croyance, si monstrueuse qu'elle pût être, n'ayant pas trouvé de défenseurs.

L'ardeur des disciples de la religion communiste tient à ce que les croyances religieuses nouvelles inspirent toujours à leurs adeptes un désir intense de détruire les vestiges de l'ancienne foi. Les nouveaux convertis n'hésitent pas, comme jadis Polyeucte, à sacrifier leur vie pour détruire les faux dieux.

À chaque période de leur histoire les hommes adoptent des religions et des philosophies à leur mesure. Bien que n'ayant généralement aucun rapport avec la réalité, elles sont fort utiles pour créer les explications dont l'esprit humain ne saurait se passer.

"S'il y a un Dieu, écrivait Pascal, il est infiniment incompréhensible... Nous sommes donc incapables de connaître, ni ce qu'il est, ni s'il est. " La science n'est pas plus avancée aujourd'hui. Elle admet cependant de plus en plus que si les Dieux existent, ils n'interviennent jamais dans la vie des êtres. 
Croire qu'on doit croire, c'est déjà croire.

\section{IV - La raison et la foi}

$\underline{\text { Retour à la table des matières }}$

L'absurdité d'un rêve ne choque pas plus le rêveur que l'absurdité d'une croyance religieuse ou politique ne choque le croyant. Rêves et croyances ont ce caractère commun d'échapper entièrement au pouvoir de la raison.

L'irrésistible force des croyances religieuses et l'impuissance de la raison sur elles apparaissent clairement quand on voit de grands génies comme Descartes et Pascal accepter des dogmes religieux qui depuis longtemps n'étaient plus défendables. Quand Newton, par exemple, dissertait sur les divagations de l'Apocalypse, sa raison était évidemment paralysée par des influences mystiques qui la dominaient entièrement.

Au point de vue de la raison pure, toutes les croyances religieuses, depuis l'adoration du serpent jusqu'à celle d'Allah, ont une valeur sensiblement égale, parce qu'elles dérivent d'illusions psychologiques identiques.

Si les croyants pouvaient se préoccuper de la valeur rationnelle de leurs croyances, il n’y aurait bientôt plus de croyants.

Le croyant refusant de raisonner sa foi pour ne pas la perdre est victime d'une crainte très chimérique. Une croyance n'est détruisible par la raison que lorsqu'elle était près de mourir dans l'âme du croyant.

Pour concevoir avec quelle lenteur la raison agit sur les croyances, il faut se rappeler que, pendant plusieurs siècles, des milliers d'hommes furent brûlés vifs à la suite de leurs relations supposées avec le diable.

Prétendre détruire une religion en persécutant ses disciples, comme le firent si longtemps les radicaux en France, montre à quel point les fondements psychologiques des croyances restent méconnus.

“C'est le cœur qui sent Dieu et non la raison ”, disait Pascal. Les dieux périraient vite si les jugements du cœur étaient ceux de la raison. 
Les parfaits dévots, comme cherchait vainement à l'être Pascal et comme l'étaient réellement les solitaires de Port-Royal, mènent une existence assez misérable. L'espoir du paradis est annulé chez eux par une horrible peur de l'enfer où ils redoutent de brûler toujours.

Une des erreurs du catholicisme moderne fut de ne pas avoir compris à quel point il devenait inacceptable en confinant à parler de supplices éternels dans un enfer sans espoir.

On peut se demander où en seraient aujourd'hui les civilisations, si l'humanité avait consacré à la poursuite de vérités scientifiques une faible partie des efforts accomplis pour obéir aux volontés de divinités imaginaires. Mais peut-être ces illusoires fantômes étaient-ils seuls capables de contraindre l'homme aux efforts dont tous ses progrès naquirent.

La conversion à une religion nouvelle est généralement instantanée, mais il lui faut des siècles pour s'implanter définitivement dans les âmes et des siècles encore pour en disparaître. Malgré la longue existence du christianisme, le paganisme grécolatin n'est pas complètement mort puisque l'art sous toutes ses formes en reste imprégné.

Les chrétiens qualifiant d'absurde l'adoration du crocodile par les Égyptiens ou du serpent par les Hindous ne se doutent pas que leurs descendants jugeront aussi absurde l'adoration d'un Dieu jugeant nécessaire de laisser crucifier son fils pour racheter une désobéissance à ses ordres.

Alors même que les civilisations futures n'accepteraient que la science pour guide, elles auraient cependant intérêt à construire encore des cathédrales, des mosquées, des pagodes, où les hommes se solidariseraient un peu en méditant sur les forces mystérieuses dont ils sont enveloppés et que personnifiaient leurs anciens Dieux.

Au point de vue purement rationnel le laboratoire est évidemment supérieur à la caserne et à l'église, mais il s'écoulera probablement bien des siècles avant qu'il soit possible de renoncer à l'église et à la caserne.

Le vrai miracle du Christianisme est d'avoir pu faire accepter pendant vingt siècles à des esprits capables de raisonner la prodigieuse légende d'un Dieu condamnant son fils à un dégradant supplice et fabriquant un enfer éternel pour y punir ses créatures. 
Une des forces du Christianisme aux États-Unis est d'être uniquement envisagé au point de vue de son utilité sociale sans se préoccuper de. la part de vérité ou d'erreur qu'il contient.

Les créateurs des dieux ont donné à l'homme la précieuse espérance, mais en échange ils l'enfermèrent pendant des siècles dans une prison d'ignorance et d'erreurs.

Il n'est guère d'idéal qui ne contienne une forte part d'illusions et cependant aucun peuple n'a pu prospérer sans l'influence d'un idéal.

L'expression " je ne veux pas croire que " dérive d'une erreur psychologique. Ce n'est jamais par un effort de la volonté consciente que l'on croit ou que l'on ne croit pas. 
Les incertitudes de l'heure présente

\section{Chapitre X}

\section{Visions philosophiques du monde}

\section{I - Conceptions philosophiques du monde}

$\underline{\text { Retour à la table des matières }}$

Le monde est-il réel ou irréel, fini ou infini, créé ou incréé, éphémère ou éternel ? La science n'entrevoit pas le moment où elle pourra répondre à une seule de ces questions.

L'impossibilité de découvrir la nature intime de la vie de la matière et de la force montre que l'esprit humain reste encore confiné dans la connaissance des effets sans pouvoir remonter à leurs causes.

La physique est la science du réel, la métaphysique celle de l'irréel, mais jusqu'ici le monde a été beaucoup plus guide par l'irréel que par le réel. La métaphysique reste donc la grande théoricienne du monde. 
Vouloir comprendre trop vite est se condamner à ne jamais comprendre.

Les grands progrès résultent de la substitution du quantitatif au qualitatif. Des instruments de mesure comme la balance, le galvanomètre et le thermomètre, ont plus modifié nos pensées et nos conditions d'existence que toutes les dissertations philosophiques.

La vie d'un pur esprit, c'est-à-dire d'un être dépourvu d'organes des sens et, par conséquent, de sensibilité, serait fort misérable. Homère l'avait pressenti quand il fait dire à l'ombre d'Achille évoquée par Ulysse : “Je préférerais n'être qu' un pauvre laboureur sur terre que roi dans l'empire des ombres."

Lorsque les êtres qui nous entourent semblent disparaître, ils ne font en réalité que changer de forme. L'évanouissement total étant scientifiquement impossible, les éléments fondamentaux des êtres ne sont concevables que sous un aspect éternel.

Le passé est formé d'événements définitivement fixés. L'éphémère présent devient rapidement un passé, fixé à son tour. L'avenir se compose d'éléments non fixés encore, mais déterminés déjà par l'état présent.

Le présent résultant du passé qui l'a précédé, on peut dire que le présent se compose surtout de passé.

Le temps ne nous est accessible que sous forme de changement : changement de position d'un astre, de l'aiguille d'un cadran ou encore des êtres qui nous entourent. Les Cieux éternels des religions ne pouvant changer, le temps y serait nécessairement inconnu.

Certains métaphysiciens contestent la réalité du temps, mais ils ne sauraient nier qu'une loi universelle oblige les êtres à naître, grandir, décliner et mourir. C'est à cette loi du changement que nous pouvons donner le nom de temps.

Vivre c'est changer. Le changement est l'âme des choses.

La façon d'envisager la vie se transforme dès qu'au lieu de la considérer à travers notre personnalité éphémère nous l'envisageons à travers la personnalité collective durable de la race. Xerxès était attristé à l'idée qu'aucun homme de son immense armée ne survivrait dans un siècle. Revenu au bout de ce siècle et retrouvant la même armée formée d'hommes aussi jeunes, possédant les mêmes visages, il eût compris que la mort n'est pas définitive puisque les défunts d'un jour revivent bientôt dans leurs descendants. 
Si tous les phénomènes physiques, chimiques et biologiques dépassant notre compréhension devaient être qualifiés de surnaturels, il n'y aurait guère que du surnaturel dans le monde.

Certains philosophes admettent que le monde perçu par nos sens est une création artificielle de ces sens. Il importe peu que le monde observé soit un monde artificiel déformé puisque l'ensemble des déformations qui le constituent est soumis à des lois dont l'observation vérifie la constance.

Aimez-vous les uns les autres, disent les religions. Dévorez-vous les uns les autres, prescrit la nature. Si l'homme n'avait pas, comme tous les êtres, respecté les prescriptions de la nature, il vivrait encore au fond des cavernes sans ébauche de civilisation.

La vie des plus grands génies semble avoir pour la nature juste autant d'importance que celle d'une colonie de microbes ou d'une fourmilière.

La compréhension des causes premières semble si au-dessus de notre intelligence qu'un être supérieur venu d'un monde lointain pour nous les expliquer n'y réussirait probablement pas mieux que si nous voulions apprendre l'algèbre à un singe.

Le savant est souvent embarrassé pour déterminer les causes d'un. phénomène. L'ignorant ne l'est jamais.

L'avenir est contenu dans le présent comme le chêne l'est dans le gland. Le temps fait sortir le chêne du gland, mais ne le crée pas.

\section{II - La vérité et la certitude}

$\underline{\text { Retour à la table des matières }}$ tudes.

Les hommes se passent facilement de vérités. Ils n’ont jamais vécu sans certi-

Les croyances les plus visiblement absurdes aux yeux de la raison sont généralement celles qui font naître le plus de certitudes. Pendant de longs siècles les hommes eurent la certitude que Moloch exigeait qu'on lui immolât des enfants et que Jéhovah condamnait à des supplices éternels les créatures n'obéissant pas à ses lois. Les communistes modernes possèdent des certitudes du même ordre. 
L'ère des grands progrès scientifiques qui transformèrent les civilisations naquit seulement lorsque l'homme réussit à distinguer la vérité de la certitude.

Les illusions tenues pour des certitudes furent parfois aussi fécondes que des vérités. La raison construit des gares et des laboratoires, mais elle n'eût jamais fait surgir du néant les pyramides, les mosquées, les cathédrales et toutes les merveilles qui ornent nos civilisations.

Les vérités scientifiques sont des vérités universelles. Les certitudes religieuses ou politiques tenues pour des vérités sont généralement des convictions transitoires issues de passions et de sentiments n'ayant aucun élément rationnel pour soutien.

Les certitudes chimériques étant chargées d'inépuisables espérances, la froide raison restera toujours impuissante contre elles.

Les philosophes qui, jadis, considéraient comme vérité toute opinion acceptée par le consentement universel confondaient la vérité et la certitude. L'adhésion universelle crée des certitudes. La science seule édifie des vérités.

Depuis l'origine des âges les adorateurs des divinités peuplant les cieux crurent posséder des certitudes. Il fallut des siècles de réflexion pour découvrir qu'il n'existait aucune trace de vérité dans ces certitudes.

Alors que les certitudes religieuses finissent toujours par périr, les vérités scientifiques restent éternelles. Celles énoncées par Archimède et Euclide gardent la même valeur qu'il y a 2000 ans.

Dans le domaine de la science, les choses ont une valeur réelle indépendante des opinions. Dans celui des croyances, elles n'ont guère d'autre valeur que l'idée qu'on s'en fait.

Il faut parfois longtemps pour qu'une vérité démontrée devienne une vérité acceptée.

Les faits scientifiquement démontrés restent immuables mais leur explication varie avec les progrès de la connaissance. Les théories de Darwin et de Pasteur sont déjà dépassées. L'atome, jadis miracle de simplicité, est devenu miracle de complexité.

Dans les sciences s'adressant à l'intelligence, la forme offre peu d'importance. Elle est essentielle au contraire dans la littérature, qui s'adresse surtout aux sentiments. D'illustres écrivains n'eurent sur la vie les sociétés, les conflits des peuples, etc., que de puériles conceptions. On les admire très justement pourtant, comme on admire la forme d'une statue sans s'occuper de la matière qui la compose. 
Pourquoi les créateurs de légendes n'ont-ils jamais imaginé un monde peuplé d'êtres dépourvus d'illusion? Sans doute parce que la vie de tels êtres ne serait pas concevable.

Les réalités scientifiques les plus solides contiennent toujours, cependant, une part notable d'illusions. Les progrès de la science consistent surtout à la réduire.

Si une conviction d'origine sentimentale est toujours plus forte qu'une conviction d'origine rationnelle, c'est que les sentiments dominent facilement la raison, alors que la raison a peu d'action sur les sentiments.

\section{III - Les lois de la vie}

$\underline{\text { Retour à la table des matières }}$

L'enfant venant à la lumière est déjà très vieux, puisqu'il représente la synthèse d'un immense passé. Son âme individuelle se trouve constituée par une accumulation de résidus d'âmes ancestrales.

Il est aussi exact d'affirmer qu'on ne revoit jamais le même être que de constater, avec Héraclite, qu'on ne se baigne pas deux fois dans le même fleuve. Cette seconde assertion est évidente, alors qu'il a fallu tous les progrès de la physiologie pour établir la première.

La vie apparaît aujourd'hui comme une puissance directrice chargée de passé, capable d'utiliser toutes les forces de la nature mais ne pouvant être créée par elles. Jusqu'ici la vie n'est sortie que de la vie. Elle seule peut obliger la microscopique cellule par laquelle débutent tous les êtres à subir les transformations nécessaires pour devenir un homme, un éléphant ou un chêne. L'impuissance de la science à interpréter ce mystère marque nettement les limites de notre intelligence.

Sur toute l'échelle des êtres, les supériorités exceptionnelles représentent des monstruosités. Pour cette raison, sans doute, la nature ramène leurs descendants au niveau moyen de l'espèce dont ils font partie.

Pendant les centaines de siècles qui précédèrent l'apparition de l'homme, tous les êtres vivaient fort bien sans posséder notre raison. Affirmer qu'ils étaient guidés par 
d'aveugles instincts, c'est simplement constater l'état rudimentaire de notre psychologie.

Dans tous les actes de la vie organique, les choses se passent comme s'il existait des modes de connaissance parfois très supérieurs, parfois inférieurs à notre intelligence, mais toujours fort différents de cette intelligence.

La vie est sans doute aussi indestructible que les autres forces. Si donc, comme tout semble le prouver, la personnalité est entièrement détruite par la mort, les éléments de cette personnalité servent à former de nouveaux êtres. C'est donc la vie collective et non la vie individuelle qui serait éternelle.

La lutte pour l'existence et l'aptitude à s'adapter aux variations de milieu font survivre et évoluer les mieux doués des êtres. L'hérédité transmet les changements ainsi acquis. Malgré de patients efforts, la science ne peut rien ajouter à ces bien sommaires notions sur les origines et l'évolution des êtres vivants. La partie essentielle de ces phénomènes nous échappe entièrement.

La mort intellectuelle commence dès que les opinions deviennent trop fixées pour changer. L'homme, même resté jeune, entre alors dans le domaine des morts. Le présent et l'avenir ne sont plus concevables pour lui qu'enveloppés de passé.

Si les forces qui ont fait surgir la vie de l'inerte matière sont douées d'intelligence, cette intelligence paraît dominée par d'aveugles nécessités lui ôtant toute liberté. Ce n'est pas assurément à la suite de raisonnements savants que furent créés tant de malfaisants microbes. Des raisons que ne comprend pas encore notre raison et où l'intelligence, telle que nous la connaissons, ne saurait jouer aucun rôle, semblent avoir dirigé l'évolution des êtres.

Pour les forces inexpliquées qui firent sortir la vie de la matière, et après des entassements de siècles surgir la pensée de la vie, tous les êtres sont égaux. L'existence du plus pernicieux microbe est aussi protégée par la nature que celle des autres êtres.

\section{IV - La sagesse et le bonheur}

$\underline{\text { Retour à la table des matières }}$

Unanimes pour admettre que le bonheur constitue le but principal de la vie, les hommes furent rarement d'accord sur les moyens de l'obtenir. Suivre aveuglément ses passions? Elles procurent moins de joie que de douleur. - Se guider d'après la raison? Ses clartés sont bien incertaines. - Obéir aux ordres des dieux ? Ils se taisent depuis longtemps. - S'adapter simplement aux nécessités de son milieu semble le plus sage. 
On ne gagne pas beaucoup à trop réfléchir sur la destinée. La vraie philosophie consiste peut-être à traverser la vie avec la sérénité tranquille de l'animal broutant l'herbe du sentier qui le mène à l'abattoir.

Le même animal deviendrait fort misérable s'il soupçonnait l'existence de l'abattoir.

Un des meilleurs moyens d'être heureux consiste à croire qu'on l'est réellement ou qu'on le sera un jour. Les religions créant cette certitude devaient pour cette seule raison jouer un rôle important dans la vie des peuples.

Une course trop rapide au bonheur n'est souvent qu'une course au malheur.

Il est utile de penser quelquefois. Pour rester heureux, on ne doit pas trop penser.

L'espérance de posséder les choses rend-elle plus heureux que la possession de ces choses ? Répondre à cette question impliquerait la connaissance d'un thermomètre du bonheur.

Se nourrir, se reproduire et s'entre-détruire, furent les principales occupations des peuples depuis les origines de l'histoire. Rien n'indique encore que leur existence puisse être différemment orientée.

Dans le monde physique comme dans le monde moral règne une dualité, loi fondamentale des phénomènes. Attractions et répulsions du monde physique deviennent plaisir et douleur, haine et amour du monde moral.

La hardiesse sans jugement est dangereuse ; le jugement sans hardiesse, inutile.

Rien ne sert d'agir si une idée directrice n'oriente pas utilement la volonté d'agir.

Savoir sans vouloir ne crée pas de pouvoir.

La vieillesse représente souvent une forme peu atténuée de la servitude.

Dans les relations entres individus et entre peuples la méfiance est nécessaire, mais jusqu' au jour de sa justification elle doit rester expectante et non agissante.

C'est surtout dans le déroulement des événements que le rigoureux enchaînement qualifié de fatalité joue son rôle. 
L'héroïsme peut sauver un peuple dans les circonstances difficiles, mais c'est l'accumulation journalière de petites vertus qui détermine sa grandeur.

L'injustice dont on profite devient vite de la justice.

\section{V - L’imprévisible et la région des causes}

$\underline{\text { Retour à la table des matières }}$

L'imprévisible domine l'Histoire. La bataille de la Marne, l'intervention américaine, la trahison russe, la débâcle allemande, constituent une série d'événements qu'aucun cerveau ne pouvait prévoir. Le pessimisme actuel résulte en partie de ce que les peuples se sentent enveloppés d'imprévisibles dangers.

Les Allemands furent vaincus dans la dernière guerre par l'immense part d'imprévisible que les phénomènes sociaux contiennent. Alliances, armements, tout avait été si minutieusement préparé que le triomphe de l'Allemagne paraissait certain. Elle n'aboutit pourtant qu'à une écrasante défaite.

Dans tous les phénomènes scientifiques ou sociaux les limites du prévisible sont vite atteintes. Les astronomes eux-mêmes ne prévoient que des faits très simples. Dès que les phénomènes se compliquent un peu, toute prévision leur échappe. C'est ainsi que la détermination des trajectoires de trois astres s'influençant réciproquement reste impossible.

Dans les phénomènes sociaux, la complexité des causes empêche généralement la prévision des effets.

Le calcul des probabilités qui permet de prédire certains phénomènes, tels que le chiffre des décès à un âge donné pour un pays donné, s'applique uniquement à des faits très répétés et non à des cas isolés. Aux événements collectifs seuls les prévisions sont applicables.

L'ignorance de la véritable raison des choses constitua toujours une source fréquente de luttes sociales et internationales.

C'est dans l'âme d'un peuple, beaucoup plus que dans les événements extérieurs, qu'il faut chercher les causes de sa destinée. Rome déclina quand, sous l'influence de croyances nouvelles et d'infiltrations répétées d'étrangers, son âme nationale se trouva désagrégée. 
Les véritables causes des événements échappent toujours lorsque, au lieu de rechercher leurs sources lointaines, on se préoccupe seulement de leurs origines immédiates. Beaucoup des faits de la grande guerre restent inexpliqués pour cette seule raison.

Les idées fixes rendent impossible la perception des réalités les plus visibles. Bien voir est souvent aussi difficile que prévoir.

Beaucoup d'effets visibles restent incompréhensibles parce qu'ils constituent l'extériorisation de causes invisibles, inaccessibles.

Jamais il ne fut aussi difficile qu'aujourd'hui de pressentir l'orientation prochaine de l'histoire. Certaines découvertes comme celles des forces motrices issues de la houille et du pétrole ont, sur la vie des peuples, une influence beaucoup plus considérable que celle exercée jadis par les conflits religieux ou les ambitions des rois.

Parmi les milliers d'hommes aspirant à établir le règne du droit et de la justice, combien en est-il capables de définir le droit et de comprendre la justice ?

Les événements seraient interprétés de façon bien différente si, pour les juger, l'esprit et le cœur utilisaient la même mesure.

FIN 